\title{
Multiple-attribute decision-making based on picture fuzzy Archimedean power Maclaurin symmetric mean operators
}

\author{
Yuchu Qin', Xiaolan Cui'2, Meifa Huang1,*, Yanru Zhong ${ }^{3}$, Zhemin Tang1, Peizhi Shi ${ }^{4}$ \\ 1 School of Mechanical and Electrical Engineering, Guilin University of Electronic Technology, Guilin 541004, PR China \\ 2 School of Management, Huazhong University of Science and Technology, Wuhan 430074, PR China \\ 3 Guangxi Key Laboratory of Intelligent Processing of Computer Images and Graphic, Guilin University of Electronic Technology, Guilin \\ 541004, PR China \\ 4 School of Computing and Engineering, University of Huddersfield, Huddersfield HD1 3DH, United Kingdom
}

\begin{abstract}
In this paper, a novel multiple attribute decision making method based on a set of Archimedean power Maclaurin symmetric mean operators of picture fuzzy numbers is proposed. The Maclaurin symmetric mean operator, power average operator, and operational rules based on Archimedean T-norm and T-conorm are introduced into picture fuzzy environment to construct the aggregation operators. The formal definitions of the aggregation operators are presented. Their general and specific expressions are established. The properties and special cases of the aggregation operators are respectively explored and discussed. Using the presented aggregation operators, a method for solving the multiple attribute decision making problems based on picture fuzzy numbers is designed. The method is illustrated through example and experiments and validated by comparisons. The results of the comparisons show that the proposed method is feasible and effective that can provide the generality and flexibility in aggregation of values of attributes and consideration of interactions among attributes and the capability to lower the negative effect of biased attribute values on the result of aggregation.
\end{abstract}

Keywords: Multiple attribute decision making; Picture fuzzy set; Aggregation operator; Maclaurin symmetric mean operator; Power average operator; Archimedean T-norm and T-conorm

\section{Introduction}

Multiple attribute decision making (MADM) is the process of finding the best option through comprehensively assessing the values of multiple attributes of all options. There are two essential tasks in this process. One task is to describe the values of attributes and the other task is to fuse the described values to determine the best option. One of the most important tools used in the first task is fuzzy set. Among the existing different types of fuzzy sets (Bustince et al. 2016), the fuzzy set (FS) presented by Zadeh (1965) is a classic type of fuzzy sets which leverages a degree of positive membership $\mu(0 \leq \mu \leq 1)$ to describe the degree of satisfaction. It is sufficient for fuzzy information description in some practical applications (Chen et al. 2009, 2012; Chen and Niou 2011; Chen and Chen 2014; Chen and Adam 2017; Castillo et al. 2019). However, FS is insufficient to express the fuzzy information consisting of the degrees of satisfaction, dissatisfaction, and hesitancy.

In response to this limitation, Atanassov (1986) extended FS and presented the intuitionistic fuzzy set (IFS), which provides a degree of positive membership $\mu$ and a degree of negative membership $v(0 \leq \mu \leq 1 ; 0 \leq v \leq 1 ; 0 \leq \mu+v \leq 1)$. The two degrees can respectively quantify the degrees of satisfaction and dissatisfaction, and therefore the degree of hesitancy is indirectly quantified by $1-\mu-v$. Due to such capability, IFSs have been widely applied to describe attribute values in MADM. Various research topics regarding IFSs in MADM, such as calculus for IFSs (Lei and Xu 2016, 2017; Ai and Xu 2018), intuitionistic preference relations (Liao and Xu 2014; Liao et al. 2015; Zhang and Pedrycz 2017a, 2018; Zhang et al. 2020), operations for IFSs (Jamkhaneh and Garg 2018; Dutta 2019; Dutta and Saikia 2019), information measures for IFSs (Chen et al. 2016a; Garg and Kumar 2018, 2019; Song et al. 2019; Tan et al. 2020), aggregation operators (AOs) of intuitionistic fuzzy numbers (IFNs) for MADM (Xu and Yager 2011; Chen and Chang 2016; Liu and Chen 2017; Liu and Tang 2018; Zhang et al. 2019; Seikh and Mandal 2019; Liu et al. 2020), and MADM methods based on IFSs (Wang and Zhang 2013; Chen et al. 2016b; Garg 2017a; Zhang and Pedrycz 2017b; Kumar and Garg 2018; Rani et al. 2019; Zeng et al. 2019), have received widespread attention.

Even though IFS has showed great capability in MADM, it cannot be used to describe more complex fuzzy information. IFS does not provide an approach to express the fuzzy information including the degree of neutrality. Aiming at this shortcoming, Hinde et al. (2007) introduced the theory of picture fuzzy set (PFS). A PFS extends an IFS with a degree of neutral membership $\eta$. Obviously, PFS is the generalisation of FS and IFS, since a PFS will reduce to a FS when its $\eta=v=0$ and will become an IFS if its $\eta=0$. It is obvious that PFSs have the greatest expressive capability compared to FSs and IFSs.

\footnotetext{
* Corresponding author.

E-mail address: meifahuang@yeah.net (M. Huang)
} 
Because of this, PFSs and their application in MADM have also received a lot of attention. A variety of related research topics, such as correlation coefficients of PFSs (Singh 2015), distance measure of PFSs (Son 2016), picture fuzzy clustering (Thong 2016), application of PFSs in decision making (Wang et al. 2018; Ju et al. 2019), AOs of picture fuzzy numbers (PFNs) for MADM (Garg 2017b; Wei 2017, 2018; Wei et al. 2018a; Zhang et al. 2018; Jana et al. 2019; Xu et al. 2019), MADM methods based on PFSs (Wang et al. 2018a; Liang et al. 2018), and extensions of PFSs (Wei et al. 2018b; Mahmood et al. 2019; Khalil et al. 2019) are gaining importance.

MADM problems are generally solved using traditional methods or AOs. AOs are capable of solving MADM problems in a more effective way since they can produce summary values and rankings of options, and traditional methods only output rankings (Liu and Liu 2018; Liu and Wang 2018; Qin et al. 2019a, 2020a). So far, a number of MADM methods based on AOs of PFNs have been presented, such as the methods presented by Wei (2017), Garg (2017b), Wei (2018), Jana et al. (2019), Wei et al. (2018a), Zhang et al. (2018), and Xu et al. (2019). The AOs of PFNs on which these methods are based are listed in Table 1. To the best of the knowledge, there is not yet a method that has flexibility in the aggregation of values of attributes and generality in the handling of interactions of attributes and meanwhile can lower the negative effect of biased values of attributes.

Table 1. The AOs of PFNs on Which the Seven MADM Methods Are Based

\begin{tabular}{lll}
\hline \hline Method & AOs of PFNs & \\
\hline Wei (2017) & Weighted averaging (WA); Weighted geometric (WG); Ordered WA (OWA); Ordered WG (OWG); & Operation \\
& Hybrid averaging (HA); Hybrid geometric (HG) & Algebraic \\
Garg (2017b) & Archimedean WA; Archimedean OWA; Archimedean HA & Archimedean \\
Wei (2018) & Hamacher WA, OWA, HA, WG, OWG, HG, correlated averaging (CA) and correlated geometric (CG); & \\
& Induced Hamacher OWA, OWG, CA and CG; & Hamacher \\
& Hamacher weighted prioritised average, prioritised geometric, power average and power geometric & \\
Jana et al. (2019) & Dombi WA; Dombi OWA; Dombi HA; Dombi WG; Dombi OWG; Dombi HG & Dombi \\
Wei et al. (2018a) & Weighted Heronian mean & Algebraic \\
Zhang et al. (2018) & Dombi weighted Heronian mean; Dombi weighted dual Heronian mean & Dombi \\
Xu et al. (2019) & Weighted Muirhead mean, Weighted dual Muirhead mean & Algebraic \\
\hline \hline
\end{tabular}

In practical MADM problems, the preferences of decision makers usually change dynamically and various interactions always exist among different considered attributes. To generate reasonable results for these problems, the used AOs should be general and flexible enough to capture the preferences and interactions when aggregating the values of attributes (Liu and Wang 2019). Among the existing AOs of PFNs, the Archimedean WA, Archimedean OWA, and Archimedean HA operators in (Garg 2017b) can provide flexibility in the aggregation of values of attributes. But they are only applicable for the situation where all of the considered attributes are independent of each other. The weighted Heronian mean operator in (Wei et al. 2018a) and the Dombi weighted Heronian mean and Dombi weighted dual Heronian mean operators in (Zhang et al. 2018) can work normally under the condition that there are no interactions among attributes or there are interactions between two attributes. But they could produce unreasonable results when there are interactions among multiple attributes. The weighted Muirhead mean and weighted dual Muirhead mean operators in (Xu et al. 2019) can make up for this deficiency. But they are not general and flexible enough for aggregating attribute values. Besides, the attribute values are mostly evaluated by experts. The absolute objectivity of this way is usually difficult to be ensured. This means that a few experts will provide some biased attribute values (Liu and Liu 2017). To obtain reasonable aggregation results under this circumstance, it is required to lower the negative impact of biased values of attributes. However, none of the existing AOs of PFNs have such capability. Based on the analysis above, the motivations of this paper are outlined as follows:

(1) To construct a flexible AO of PFNs, Archimedean T-norm and T-conorm (ATT) (Klement et al. 2000; Xia et al. 2012) are introduced into PFSs. The ATT operations are important tools for generalising logical conjunction and disjunction to fuzzy logic. They can be used to develop versatile rules for the operations between fuzzy numbers. The AOs using such operational rules are flexible (Liu and Wang 2019; Zhong et al. 2019a; Qin et al. 2019b, 2020b).

(2) To make the AO general in capturing the interactions of attributes, Maclaurin symmetric mean (MSM) operator (Maclaurin 1729) is chosen as its core component. The MSM operator, a generalisation of the arithmetic average (AA) operator, Bonferroni mean (BM) operator, and geometric average (GA) operator, is a general AO for describing the interactions of the aggregated arguments. It is applicable for the situations where there are no interactions among all arguments, where there are interactions between two arguments, and where there are interactions among multiple arguments.

(3) To make the AO capable to reduce the negative effect of unduly high or unduly low attribute values on the aggregation result, power average (PA) operator (Yager 2001) is combined with the MSM operator of PFNs under the ATT operations. The PA operator is an AO which can assign weights to the aggregated arguments via computing the degrees of support be- 
tween the arguments. This makes it capable to reduce the negative effect of unreasonable argument values (Liu et al. 2018; Teng et al. 2018; Zhong et al. 2019b; Qin et al. 2020c).

In a word, the objective of the paper is to present a set of Archimedean power MSM operators of PFNs for solving the MADM problems based on PFNs. This objective is achieved via combining the MSM operator and the PA operator under the ATT operations in the context of PFSs. The major contribution of the paper is the development of an MADM method based on picture fuzzy Archimedean power MSM operators. This method can provide the generality in aggregation of values of attributes, the flexibility in handling of interactions among attributes, and the capability to lower the negative impact of biased attribute values.

The rest of the paper is organised as follows. A brief introduction of some basic concepts is provided Section 2. Section 3 describes the details of the presented operators. The specific process of the proposed method is described in Section 4. Section 5 demonstrates and evaluates the proposed method. Section 6 ends the paper with a conclusion.

\section{Preliminaries}

\subsection{PFS Theory}

PFS can be seen as the generalisation of FS (Zadeh 1965) and IFS (Atanassov 1986). Its formal definition is given by Hinde et al. (2007):

Definition 1. A PFS $S$ in a finite domain of discourse $X$ is $S=\left\{\left\langle x, \mu_{S}(x), \eta_{S}(x), v_{S}(x)\right\rangle \mid x \in X\right\}$, where $\mu_{S}: X \rightarrow[0,1]$ is the degree of positive membership of $x \in X$ to $S, \eta_{S}: X \rightarrow[0,1]$ is the degree of neutral membership of $x \in X$ to $S$, and $v_{S}: X \rightarrow[0$, $1]$ is the degree of negative membership of $x \in X$ to $S$, such that $0 \leq \mu_{S}(x)+\eta_{S}(x)+v_{S}(x) \leq 1$. The degree of refusal membership of $x \in X$ to $S$ is $\pi_{S}(x)=1-\mu_{S}(x)-\eta_{S}(x)-v_{S}(x)$.

A triple $\left\langle\mu_{S}(x), \eta_{S}(x), \nu_{S}(x)\right\rangle$ is called a PFN and is usually denoted as $\langle\mu, \eta, v\rangle$. Two PFNs can be compared using their score values and accuracy values. Jana et al. (2019) introduced a function to calculate the score value of a PFN and Wei (2017) introduced a function to calculate the accuracy value of a PFN:

Definition 2. Suppose $\alpha=\langle\mu, \eta, v\rangle$ is a PFN, its score value can be calculated via $S(\alpha)=0.5 \times(1+\mu-v)$.

Definition 3. Suppose $\alpha=\langle\mu, \eta, v\rangle$ is a PFN, its accuracy value can be calculated via $A(\alpha)=\mu+\eta+v$.

Based on the score and accuracy values, Wei (2017) introduced the rules for comparing two PFNs:

Definition 4. Suppose $\alpha_{1}=\left\langle\mu_{1}, \eta_{1}, v_{1}\right\rangle$ and $\alpha_{2}=\left\langle\mu_{2}, \eta_{2}, v_{2}\right\rangle$ are two PFNs, $S\left(\alpha_{1}\right)$ and $S\left(\alpha_{2}\right)$ are their score values, and $A\left(\alpha_{1}\right)$ and $A\left(\alpha_{2}\right)$ are their accuracy values. Then: (1) $\alpha_{1}>\alpha_{2}$ if $S\left(\alpha_{1}\right)>S\left(\alpha_{2}\right) ;(2) \alpha_{1}>\alpha_{2}$ if $S\left(\alpha_{1}\right)=S\left(\alpha_{2}\right)$ and $A\left(\alpha_{1}\right)>A\left(\alpha_{2}\right) ;(3)$ $\alpha_{1}=\alpha_{2}$ if $S\left(\alpha_{1}\right)=S\left(\alpha_{2}\right)$ and $A\left(\alpha_{1}\right)=A\left(\alpha_{2}\right)$.

The distance of two PFNs can be calculated using a distance measure of PFNs. Cuong (2014) introduced a normalised Hamming distance measure of PFNs:

Definition 5. Suppose $\alpha_{1}=\left\langle\mu_{1}, \eta_{1}, v_{1}\right\rangle$ and $\alpha_{2}=\left\langle\mu_{2}, \eta_{2}, v_{2}\right\rangle$ are two PFNs. The normalised Hamming distance between them is $d\left(\alpha_{1}, \alpha_{2}\right)=0.5\left(\left|\mu_{1}-\mu_{2}\right|+\left|\eta_{1}-\eta_{2}\right|+\left|v_{1}-v_{2}\right|\right)$.

\subsection{Operational Rules}

Based on ATT, Garg (2017b) introduced a set of operational rules of PFNs:

Definition 6. Suppose $\alpha=\langle\mu, \eta, v\rangle, \alpha_{1}=\left\langle\mu_{1}, \eta_{1}, v_{1}\right\rangle$, and $\alpha_{2}=\left\langle\mu_{2}, \eta_{2}, v_{2}\right\rangle$ are three PFNs, and $c$ and $d$ are two positive real numbers. The operations of PFNs based on $T(x, y)=\varphi^{-1}(\varphi(x)+\varphi(y))$ and $T^{\mathrm{C}}(x, y)=\psi^{-1}(\psi(x)+\psi(y))$ are defined as follows:

$$
\begin{aligned}
& \alpha_{1} \oplus \alpha_{2}=\left\langle T^{\mathrm{C}}\left(\mu_{1}, \mu_{2}\right), T\left(\eta_{1}, \eta_{2}\right), T\left(v_{1}, v_{2}\right)\right\rangle=\left\langle\psi^{-1}\left(\psi\left(\mu_{1}\right)+\psi\left(\mu_{2}\right)\right), \varphi^{-1}\left(\varphi\left(\eta_{1}\right)+\varphi\left(\eta_{2}\right)\right), \varphi^{-1}\left(\varphi\left(v_{1}\right)+\varphi\left(v_{2}\right)\right)\right\rangle \\
& \alpha_{1} \otimes \alpha_{2}=\left\langle T\left(\mu_{1}, \mu_{2}\right), T^{\mathrm{C}}\left(\eta_{1}, \eta_{2}\right), T^{\mathrm{C}}\left(v_{1}, v_{2}\right)\right\rangle=\left\langle\varphi^{-1}\left(\varphi\left(\mu_{1}\right)+\varphi\left(\mu_{2}\right)\right), \psi^{-1}\left(\psi\left(\eta_{1}\right)+\psi\left(\eta_{2}\right)\right), \psi^{-1}\left(\psi\left(v_{1}\right)+\psi\left(v_{2}\right)\right)\right\rangle \\
& c \alpha=\left\langle\psi^{-1}(c \psi(\mu)), \varphi^{-1}(c \varphi(\eta)), \varphi^{-1}(c \varphi(v))\right\rangle \\
& \alpha^{d}=\left\langle\varphi^{-1}(d \varphi(\mu)), \psi^{-1}(d \psi(\eta)), \psi^{-1}(d \psi(v))\right\rangle
\end{aligned}
$$

\subsection{MSM Operator}

The MSM operator was introduced by Maclaurin (1729). This operator can be formally defined as:

Definition 7. Suppose $\mathrm{a}_{1}, \mathrm{a}_{2}, \ldots, \mathrm{a}_{n}$ are $n$ crisp numbers and $k$ is an integer such that $1 \leq k \leq n$. If $\left(i_{1}, i_{2}, \ldots, i_{k}\right)$ traverse all the $k$-tuple combinations of $(1,2, \ldots, n)$, then

$$
\operatorname{MSM}^{(k)}\left(a_{1}, a_{2}, \ldots, a_{n}\right)=\left(\frac{k !(n-k) !}{n !} \sum_{1 \leq i_{1}<\ldots<i_{k} \leq n} \prod_{h=1}^{k} a_{i_{h}}\right)^{1 / k}
$$

is called the MSM operator. 


\subsection{PA Operator}

The PA operator was introduced by Yager (2001). This operator can be formally defined as:

Definition 8. Suppose $\mathrm{a}_{1}, \mathrm{a}_{2}, \ldots, \mathrm{a}_{n}$ are $n$ crisp numbers, $S\left(\mathrm{a}_{i}, \mathrm{a}_{j}\right)=1-d\left(\mathrm{a}_{i}, \mathrm{a}_{j}\right)\left(i, j=1,2, \ldots, n\right.$ and $j \neq i ; d\left(\mathrm{a}_{i}, \mathrm{a}_{j}\right)$ is the distance between $\mathrm{a}_{i}$ and $\left.\mathrm{a}_{j}\right)$ is the degree of support for $\mathrm{a}_{i}$ from $\mathrm{a}_{j}$ which satisfies $\left.0 \leq S\left(\mathrm{a}_{i}, \mathrm{a}_{j}\right) \leq 1, S\left(\mathrm{a}_{i}, \mathrm{a}_{j}\right)=S\left(\mathrm{a}_{j}, \mathrm{a}_{i}\right), a_{1}\right) S\left(\mathrm{a}_{i}, \mathrm{a}_{j}\right) \geq$ $S\left(\mathrm{a}_{p}, \mathrm{a}_{q}\right)$ if $\left|\mathrm{a}_{i}, \mathrm{a}_{j}\right| \leq\left|\mathrm{a}_{p}, \mathrm{a}_{q}\right|$, and

$$
T\left(a_{i}\right)=\sum_{j=1, j \neq i}^{n} S\left(a_{i}, a_{j}\right)
$$

Then

$$
P A\left(a_{1}, a_{2}, \ldots, a_{n}\right)=\sum_{i=1}^{n}\left(\left(1+T\left(a_{i}\right)\right) a_{i}\right) / \sum_{i=1}^{n}\left(1+T\left(a_{i}\right)\right)
$$

is called the PA operator.

\section{Archimedean Power MSM Operators}

\subsection{PFAPMSM Operator}

A picture fuzzy Archimedean power MSM (PFAPMSM) operator is a power MSM operator of PFNs, in which the operations are carried out via the operational rules of PFNs based on ATT. This operator can be formally defined as:

Definition 9. Suppose $\alpha_{1}, \alpha_{2}, \ldots, \alpha_{n}\left(\alpha_{i}=\left\langle\mu_{i}, \eta_{i}, v_{i}\right\rangle, i=1,2, \ldots, n\right)$ are $n$ PFNs, $k$ is an integer which meets $1 \leq k \leq n$, $\alpha_{p} \oplus \alpha_{q}$ and $\alpha_{p} \otimes \alpha_{q}(p, q=1,2, \ldots, n$ and $p \neq q)$ and $c \alpha_{r}$ and $\alpha_{s}{ }^{d}(r, s=1,2, \ldots, n$ and $r \neq s ; c, d>0)$ are the operations of PFNs based on ATT, and $S\left(\alpha_{p}, \alpha_{q}\right)=1-d\left(\alpha_{p}, \alpha_{q}\right)\left(d\left(\alpha_{p}, \alpha_{q}\right)\right.$ is the distance between $\alpha_{p}$ and $\left.\alpha_{q}\right)$ be the degree of support for $\alpha_{p}$ from $\alpha_{q}$ which satisfies $0 \leq S\left(\alpha_{p}, \alpha_{q}\right) \leq 1, S\left(\alpha_{p}, \alpha_{q}\right)=S\left(\alpha_{q}, \alpha_{p}\right)$, and $S\left(\alpha_{p}, \alpha_{q}\right) \geq S\left(\alpha_{r}, \alpha_{s}\right)$ if $\left|\alpha_{p}-\alpha_{q}\right| \leq\left|\alpha_{r}-\alpha_{s}\right|$, and

$$
T\left(\alpha_{p}\right)=\sum_{q=1, q \neq p}^{n} S\left(\alpha_{p}, \alpha_{q}\right)
$$

If $\left(i_{1}, i_{2}, \ldots, i_{k}\right)$ traverse all the $k$-tuple combinations of $(1,2, \ldots, n)$, then

$$
\operatorname{PFAPMSM}^{(k)}\left(\alpha_{1}, \alpha_{2}, \ldots, \alpha_{n}\right)=\left(\frac{1}{C_{n}^{k}} \bigoplus_{1 \leq i_{1}<\ldots<i_{k} \leq n h} \bigotimes_{h=1}^{k}\left(\frac{n\left(1+T\left(\alpha_{i_{h}}\right)\right)}{\sum_{j=1}^{n}\left(1+T\left(\alpha_{j}\right)\right)} \alpha_{i_{h}}\right)\right)^{1 / k}
$$

is called the PFAPMSM operator.

The general expression of the PFAPMSM is constructed in the following theorem:

Theorem 1. Suppose $\alpha_{1}, \alpha_{2}, \ldots, \alpha_{n}\left(\alpha_{i}=\left\langle\mu_{i}, \eta_{i}, v_{i}\right\rangle, i=1,2, \ldots, n\right)$ are $n$ PFNs. Then

$$
\operatorname{PFAPMSM}^{(k)}\left(\alpha_{1}, \alpha_{2}, \ldots, \alpha_{n}\right)=\langle\mu, \eta, v\rangle
$$

where

$$
\begin{aligned}
& \mu=\varphi^{-1}\left(\frac{1}{k} \varphi\left(\psi^{-1}\left(\frac{1}{C_{n}^{k}} \sum_{1 \leq i_{1}<\ldots<i_{k} \leq n} \psi\left(\varphi^{-1}\left(\sum_{h=1}^{k} \varphi\left(\psi^{-1}\left(\left(n \omega_{i_{h}}\right) \psi\left(\mu_{i_{h}}\right)\right)\right)\right)\right)\right)\right)\right) \\
& \eta=\psi^{-1}\left(\frac{1}{k} \psi\left(\varphi^{-1}\left(\frac{1}{C_{n}^{k}} \sum_{1 \leq i_{1}<\ldots<i_{k} \leq n} \varphi\left(\psi^{-1}\left(\sum_{h=1}^{k} \psi\left(\varphi^{-1}\left(\left(n \omega_{i_{h}}\right) \varphi\left(\eta_{i_{h}}\right)\right)\right)\right)\right)\right)\right)\right) \\
& \nu=\psi^{-1}\left(\frac{1}{k} \psi\left(\varphi^{-1}\left(\frac{1}{C_{n}^{k}} \sum_{1 \leq i_{1}<\ldots<i_{k} \leq n} \varphi\left(\psi^{-1}\left(\sum_{h=1}^{k} \psi\left(\varphi^{-1}\left(\left(n \omega_{i_{h}}\right) \varphi\left(v_{i_{h}}\right)\right)\right)\right)\right)\right)\right)\right) \\
& \omega_{i_{h}}=\left(1+T\left(\alpha_{i_{h}}\right)\right) / \sum_{j=1}^{n}\left(1+T\left(\alpha_{j}\right)\right)
\end{aligned}
$$

and $\operatorname{PFAPMSM}^{(k)}\left(\alpha_{1}, \alpha_{2}, \ldots, \alpha_{n}\right)$ is still a PFN.

The proof of this theorem is provided in App. A. The properties of the PFAPMSM operator are stated in the following theorems:

Theorem 2 (Idempotency). Suppose $\alpha_{1}, \alpha_{2}, \ldots, \alpha_{n}\left(\alpha_{i}=\left\langle\mu_{i}, \eta_{i}, v_{i}\right\rangle, i=1,2, \ldots, n\right)$ are $n$ PFNs. If $\alpha_{i}=\alpha=\left\langle\mu_{\alpha}, \eta_{\alpha}, v_{\alpha}\right\rangle$ for all $i=1,2, \ldots, n$, then $\operatorname{PFAPMSM}^{(k)}\left(\alpha_{1}, \alpha_{2}, \ldots, \alpha_{n}\right)=\alpha=\left\langle\mu_{\alpha}, \eta_{\alpha}, v_{\alpha}\right\rangle$. 
Theorem 3 (Commutativity). Suppose $\alpha_{1}, \alpha_{2}, \ldots, \alpha_{n}\left(\alpha_{i}=\left\langle\mu_{i}, \eta_{i}, v_{i}\right\rangle, i=1,2, \ldots, n\right)$ are $n$ PFNs. If $\left(\beta_{1}, \beta_{2}, \ldots, \beta_{n}\right)$ is any permutation of $\left(\alpha_{1}, \alpha_{2}, \ldots, \alpha_{n}\right)$, then $\operatorname{PFAPMSM}^{(k)}\left(\beta_{1}, \beta_{2}, \ldots, \beta_{n}\right)=\operatorname{PFAPMSM}^{(k)}\left(\alpha_{1}, \alpha_{2}, \ldots, \alpha_{n}\right)$.

Theorem 4 (Boundedness). Suppose $\alpha_{1}, \alpha_{2}, \ldots, \alpha_{n}\left(\alpha_{i}=\left\langle\mu_{i}, \eta_{i}, v_{i}\right\rangle, i=1,2, \ldots, n\right)$ are $n$ PFNs, $\alpha^{+}=\left\langle\max \left(\mu_{i}\right), \min \left(\eta_{i}\right)\right.$, $\min \left(v_{i}\right)>$, and $\alpha^{-}=\left\langle\min \left(\mu_{i}\right), \max \left(\eta_{i}\right), \max \left(v_{i}\right)>\right.$. Then $\alpha^{-} \leq$PFAPMSM $^{(k)}\left(\alpha_{1}, \alpha_{2}, \ldots, \alpha_{n}\right) \leq \alpha^{+}$.

The proofs of these theorems are respectively provided in App.es B, C, and D. Some special cases of the PFAPMSM operator are as follows:

(1) If $k=1$, the PFAPMSM operator will become

$$
\left\langle\psi^{-1}\left(\frac{1}{n} \sum_{i=1}^{n}\left(\left(n \omega_{i}\right) \psi\left(\mu_{i}\right)\right)\right), \varphi^{-1}\left(\frac{1}{n} \sum_{i=1}^{n}\left(\left(n \omega_{i}\right) \varphi\left(\eta_{i}\right)\right)\right), \varphi^{-1}\left(\frac{1}{n} \sum_{i=1}^{n}\left(\left(n \omega_{i}\right) \varphi\left(v_{i}\right)\right)\right)\right\rangle=\operatorname{PFAPAA}\left(\alpha_{1}, \alpha_{2}, \ldots, \alpha_{n}\right)
$$

which is a picture fuzzy Archimedean power AA (PFAPAA) operator.

(2) If $k=2$, the PFAPMSM operator will become

$$
\begin{gathered}
\left\langle\varphi ^ { - 1 } \left(\frac{1}{2} \varphi\left(\psi^{-1}\left(\frac{1}{n(n-1)} \sum_{\substack{i_{1}, i_{2}=1 \\
i_{2} \neq i_{1}}}^{n} \psi\left(\varphi^{-1}\left(\varphi\left(\psi^{-1}\left(\left(n \omega_{i_{1}}\right) \psi\left(\mu_{i_{1}}\right)\right)\right)+\varphi\left(\psi^{-1}\left(\left(n \omega_{i_{2}}\right) \psi\left(\mu_{i_{2}}\right)\right)\right)\right)\right)\right)\right),\right.\right. \\
\psi^{-1}\left(\frac{1}{2} \psi\left(\varphi^{-1}\left(\frac{1}{n(n-1)} \sum_{\substack{i_{1}, i_{2}=1 \\
i_{2} \neq i_{1}}}^{n} \varphi\left(\psi^{-1}\left(\psi\left(\varphi^{-1}\left(\left(n \omega_{i_{1}}\right) \varphi\left(\eta_{i_{1}}\right)\right)\right)+\psi\left(\varphi^{-1}\left(\left(n \omega_{i_{2}}\right) \varphi\left(\eta_{i_{2}}\right)\right)\right)\right)\right)\right)\right),\right. \\
\left.\psi^{-1}\left(\frac{1}{2} \psi\left(\varphi^{-1}\left(\frac{1}{n(n-1)} \sum_{\substack{i_{1}, i_{2}=1 \\
i_{2} \neq i_{1}}}^{n} \varphi\left(\psi^{-1}\left(\psi\left(\varphi^{-1}\left(\left(n \omega_{i_{1}}\right) \varphi\left(v_{i_{1}}\right)\right)\right)+\psi\left(\varphi^{-1}\left(\left(n \omega_{i_{2}}\right) \varphi\left(v_{i_{2}}\right)\right)\right)\right)\right)\right)\right)\right)\right\rangle=
\end{gathered}
$$

$\operatorname{PFAPBM}^{(1,1)}\left(\alpha_{1}, \alpha_{2}, \ldots, \alpha_{n}\right)$

which is a picture fuzzy Archimedean power BM (PFAPBM) operator.

(3) If $k=n$, the PFAPMSM operator will become

$$
\left\langle\varphi^{-1}\left(\frac{1}{n} \sum_{i=1}^{n} \varphi\left(\psi^{-1}\left(\left(n \omega_{i}\right) \psi\left(\mu_{i}\right)\right)\right)\right), \psi^{-1}\left(\frac{1}{n} \sum_{i=1}^{n} \psi\left(\varphi^{-1}\left(\left(n \omega_{i}\right) \varphi\left(\eta_{i}\right)\right)\right)\right), \psi^{-1}\left(\frac{1}{n} \sum_{i=1}^{n} \psi\left(\varphi^{-1}\left(\left(n \omega_{i}\right) \varphi\left(v_{i}\right)\right)\right)\right)\right\rangle=
$$

$\operatorname{PFAPGA}\left(\alpha_{1}, \alpha_{2}, \ldots, \alpha_{n}\right)$

which is a picture fuzzy Archimedean power GA (PFAPGA) operator.

The specific expressions of the PFAPMSM operator are constructed as follows:

(1) If Algebraic T-norm and T-conorm are applied to Eq. (8), a picture fuzzy Archimedean Algebraic power MSM (PFAAPMSM) operator can be obtained as:

$$
\begin{aligned}
& \operatorname{PFAAPMSM} M^{(k)}\left(\alpha_{1}, \alpha_{2}, \ldots, \alpha_{n}\right)=\left\langle\left(1-\left(\prod_{1 \leq i_{1}<\ldots<i_{k} \leq n}\left(1-\prod_{h=1}^{k}\left(1-\left(1-\mu_{i_{h}}\right)^{n \omega_{i_{h}}}\right)\right)\right)^{1 / c_{n}^{k}}\right)^{1 / k},\right. \\
& \left.\left.1-\left(1-\left(\prod_{1 \leq i_{1}<\ldots<i_{k} \leq n}\left(1-\prod_{h=1}^{k}\left(1-\eta_{i_{h}}^{n \omega_{i_{h}}}\right)\right)\right)^{1 / C_{n}^{k}}\right)^{1 / k}, 1-\left(1-\left(\prod_{1 \leq i_{1}<\ldots<i_{k} \leq n}\left(1-\prod_{h=1}^{k}\left(1-v_{i_{h}}^{n \omega_{i_{h}}}\right)\right)\right)^{1 / c_{n}^{k}}\right)^{1 / k}\right)^{1 / k}\right\rangle
\end{aligned}
$$

Some special cases of this operator are as follows:

a) If $k=1$, the PFAAPMSM operator will become

$$
\left\langle 1-\prod_{i=1}^{n}\left(1-\mu_{i}\right)^{\omega_{i}}, \prod_{i=1}^{n} \eta_{i}^{\omega_{i}}, \prod_{i=1}^{n} v_{i}^{\omega_{i}}\right\rangle=\operatorname{PFPAA}\left(\alpha_{1}, \alpha_{2}, \ldots, \alpha_{n}\right)
$$

which is a picture fuzzy power AA (PFPAA) operator.

b) If $k=2$, the PFAAPMSM operator will become

$$
\left\langle\left(1-\prod_{\substack{i_{1}, i_{2}=1 \\ i_{2} \neq i_{1}}}^{n}\left(1-\left(1-\left(1-\mu_{i_{1}}\right)^{n \omega_{i_{1}}}\right)\left(1-\left(1-\mu_{i_{2}}\right)^{n \omega_{i_{2}}}\right)\right)^{\frac{1}{n(n-1)}}\right)^{1 / 2}, 1-\left(1-\prod_{\substack{i_{1}, i_{2}=1 \\ i_{2} \neq i_{1}}}^{n}\left(1-\left(1-\eta_{i_{1}}^{n \omega_{i_{1}}}\right)\left(1-\eta_{i_{2}}^{n \omega_{i_{2}}}\right)\right)^{\frac{1}{n(n-1)}}\right)^{1 / 2},\right.
$$




$$
\left.1-\left(1-\prod_{\substack{i_{1}, i_{2}=1 \\ i_{2} \neq i_{1}}}^{n}\left(1-\left(1-v_{i_{1}}^{n \omega_{11}}\right)\left(1-v_{i_{2}}^{n \omega_{i_{2}}}\right)\right)^{\frac{1}{n(n-1)}}\right)^{1 / 2}\right\rangle=\operatorname{PFPBM}^{(1,1)}\left(\alpha_{1}, \alpha_{2}, \ldots, \alpha_{n}\right)
$$

which is a picture fuzzy power BM (PFPBM) operator.

c) If $k=n$, the PFAAPMSM operator will become

$$
\left\langle\prod_{i=1}^{n}\left(1-\left(1-\mu_{i}\right)^{n \omega_{i}}\right)^{1 / n}, 1-\prod_{i=1}^{n}\left(1-\eta_{i}^{n \omega_{i}}\right)^{1 / n}, 1-\prod_{i=1}^{n}\left(1-v_{i}^{n \omega_{i}}\right)^{1 / n}\right\rangle=\operatorname{PFPGA}\left(\alpha_{1}, \alpha_{2}, \ldots, \alpha_{n}\right)
$$

which is a picture fuzzy power GA (PFPGA) operator.

(2) If Einstein T-norm and T-conorm are applied to Eq. (8), a picture fuzzy Archimedean Einstein power MSM (PFAEPMSM) operator can be obtained as:

$$
\begin{aligned}
& \operatorname{PFAEPMSM}^{(k)}\left(\alpha_{1}, \alpha_{2}, \ldots, \alpha_{n}\right)=\left\langle\left(2\left(\mu^{\prime \prime}-1\right)^{1 / k}\right) /\left(\left(\mu^{\prime \prime}+3\right)^{1 / k}+\left(\mu^{\prime \prime}-1\right)^{1 / k}\right),\right. \\
& \left.\left(\left(\eta^{\prime \prime}+3\right)^{1 / k}-\left(\eta^{\prime \prime}-1\right)^{1 / k}\right) /\left(\left(\eta^{\prime \prime}+3\right)^{1 / k}+\left(\eta^{\prime \prime}-1\right)^{1 / k}\right),\left(\left(v^{\prime \prime}+3\right)^{1 / k}-\left(v^{\prime \prime}-1\right)^{1 / k}\right) /\left(\left(v^{\prime \prime}+3\right)^{1 / k}+\left(v^{\prime \prime}-1\right)^{1 / k}\right)\right\rangle
\end{aligned}
$$

where

$$
\begin{aligned}
& \mu^{\prime \prime}=\prod_{1 \leq i_{1}<\ldots<i_{k} \leq n}\left(\left(\mu^{\prime}+3\right) /\left(\mu^{\prime}-1\right)\right)^{1 / C_{n}^{k}}, \mu^{\prime}=\prod_{h=1}^{k}\left(\left(\left(1+\mu_{i_{h}}\right)^{n \omega_{i_{h}}}+3\left(1-\mu_{i_{h}}\right)^{n \omega_{i_{h}}}\right) /\left(\left(1+\mu_{i_{h}}\right)^{n \omega_{i_{h}}}-\left(1-\mu_{i_{h}}\right)^{n \omega_{i_{h}}}\right)\right) \\
& \eta^{\prime \prime}=\prod_{1 \leq i_{1}<\ldots<i_{k} \leq n}\left(\left(\eta^{\prime}+3\right) /\left(\eta^{\prime}-1\right)\right)^{1 / c_{n}^{k}}, \eta^{\prime}=\prod_{h=1}^{k}\left(\left(\left(2-\eta_{i_{h}}\right)^{n \omega_{i_{h}}}+3 \eta_{i_{h}}^{n \omega_{i_{h}}}\right) /\left(\left(2-\eta_{i_{h}}\right)^{n \omega_{i_{h}}}-\eta_{i_{h}}^{n \omega_{i_{h}}}\right)\right) \\
& v^{\prime \prime}=\prod_{1 \leq i_{1}<\ldots<i_{k} \leq n}\left(\left(v^{\prime}+3\right) /\left(v^{\prime}-1\right)\right)^{1 / c_{n}^{k}}, v^{\prime}=\prod_{h=1}^{k}\left(\left(\left(2-v_{i_{h}}\right)^{n \omega_{i_{h}}}+3 v_{i_{h}}^{n \omega_{i_{h}}}\right) /\left(\left(2-v_{i_{h}}\right)^{n \omega_{i_{h}}}-v_{i_{h}}^{n \omega_{i_{h}}}\right)\right)
\end{aligned}
$$

(3) If Hamacher T-norm and T-conorm are applied to Eq. (8), a picture fuzzy Archimedean Hamacher power MSM (PFAHPMSM) operator can be obtained as:

$$
\begin{aligned}
\operatorname{PFAHPMSM}^{(k)}\left(\alpha_{1}, \alpha_{2}, \ldots, \alpha_{n}\right)= & \left\langle\left(\lambda\left(\mu^{\prime \prime}-1\right)^{1 / k}\right) /\left(\left(\mu^{\prime \prime}+\lambda^{2}-1\right)^{1 / k}+(\lambda-1)\left(\mu^{\prime \prime}-1\right)^{1 / k}\right),\right. \\
& \left(\left(\eta^{\prime \prime}+\lambda^{2}-1\right)^{1 / k}-\left(\eta^{\prime \prime}-1\right)^{1 / k}\right) /\left(\left(\eta^{\prime \prime}+\lambda^{2}-1\right)^{1 / k}+(\lambda-1)\left(\eta^{\prime \prime}-1\right)^{1 / k}\right), \\
& \left.\left(\left(v^{\prime \prime}+\lambda^{2}-1\right)^{1 / k}-\left(v^{\prime \prime}-1\right)^{1 / k}\right) /\left(\left(v^{\prime \prime}+\lambda^{2}-1\right)^{1 / k}+(\lambda-1)\left(v^{\prime \prime}-1\right)^{1 / k}\right)\right\rangle
\end{aligned}
$$

where $\lambda>0$ and

$$
\begin{aligned}
& \mu^{\prime \prime}=\prod_{1 \leq i_{1}<\ldots<i_{k} \leq n}\left(\left(\mu^{\prime}+\lambda^{2}-1\right) /\left(\mu^{\prime}-1\right)\right)^{1 / c_{n}^{k}}, \mu^{\prime}=\prod_{h=1}^{k} \frac{\left(\lambda+(1-\lambda)\left(1-\mu_{i_{h}}\right)\right)^{n \omega_{i_{h}}}+\left(\lambda^{2}-1\right)\left(1-\mu_{i_{h}}\right)^{n \omega_{i_{h}}}}{\left(\lambda+(1-\lambda)\left(1-\mu_{i_{h}}\right)\right)^{n \omega_{i_{h}}}-\left(1-\mu_{i_{h}}\right)^{n \omega_{i_{h}}}} \\
& \eta^{\prime \prime}=\prod_{1 \leq i_{1}<\ldots<i_{k} \leq n}\left(\left(\eta^{\prime}+\lambda^{2}-1\right) /\left(\eta^{\prime}-1\right)\right)^{1 / C_{n}^{k}}, \eta^{\prime}=\prod_{h=1}^{k}\left(\left(\left(\lambda+(1-\lambda) \eta_{i_{h}}\right)^{n \omega_{i_{h}}}+\left(\lambda^{2}-1\right) \eta_{i_{h}}^{n \omega_{i_{h}}}\right) /\left(\left(\lambda+(1-\lambda) \eta_{i_{h}}\right)^{n \omega_{i_{h}}}-\eta_{i_{h}}^{n \omega_{i_{h}}}\right)\right) \\
& v^{\prime \prime}=\prod_{1 \leq i_{1}<\ldots<i_{k} \leq n}\left(\left(v^{\prime}+\lambda^{2}-1\right) /\left(v^{\prime}-1\right)\right)^{1 / C_{n}^{k}}, v^{\prime}=\prod_{h=1}^{k}\left(\left(\left(\lambda+(1-\lambda) v_{i_{h}}\right)^{n \omega_{i_{h}}}+\left(\lambda^{2}-1\right) v_{i_{h}}^{n \omega_{i_{h}}}\right) /\left(\left(\lambda+(1-\lambda) v_{i_{h}}\right)^{n \omega_{i_{h}}}-v_{i_{h}}^{n \omega_{i_{h}}}\right)\right)
\end{aligned}
$$

(4) If Frank T-norm and T-conorm are applied to Eq. (8), a picture fuzzy Archimedean Frank power MSM (PFAFPMSM) operator can be obtained as:

$$
\begin{array}{r}
\operatorname{PFAFPMSM}^{(k)}\left(\alpha_{1}, \alpha_{2}, \ldots, \alpha_{n}\right)=\left\langle\log _{\varepsilon}\left(1+\left(\varepsilon^{\mu^{\prime \prime \prime}}-1\right)^{1 / k} /(\varepsilon-1)^{1 / k-1}\right),\right. \\
1-\log _{\varepsilon}\left(1+\left(\varepsilon^{1-\eta^{\prime \prime \prime}}-1\right)^{1 / k} /(\varepsilon-1)^{1 / k-1}\right), \\
\left.1-\log _{\varepsilon}\left(1+\left(\varepsilon^{1-v^{\prime \prime \prime}}-1\right)^{1 / k} /(\varepsilon-1)^{1 / k-1}\right)\right\rangle
\end{array}
$$

where $\varepsilon>1$ and

$$
\begin{aligned}
& \mu^{\prime \prime \prime}=1-\log _{\varepsilon}\left(1+(\varepsilon-1) / \prod_{1 \leq i_{1}<\ldots<<_{k} \leq n}\left((\varepsilon-1) /\left(\varepsilon^{1-\mu^{\prime \prime}}-1\right)\right)^{1 / C_{n}^{k}}\right), \mu^{\prime \prime}=\log _{\varepsilon}\left(1+(\varepsilon-1) / \prod_{h=1}^{k}\left((\varepsilon-1) /\left(\varepsilon^{\mu^{\prime}}-1\right)\right)\right), \\
& \mu^{\prime}=1-\log _{\varepsilon}\left(1+\left(\left(\varepsilon^{1-\mu_{i_{h}}}-1\right)^{n \omega_{i_{h}}} /(\varepsilon-1)^{n \omega_{i_{h}}-1}\right)\right),
\end{aligned}
$$




$$
\begin{aligned}
& \eta^{\prime \prime \prime}=\log _{\varepsilon}\left(1+(\varepsilon-1) / \prod_{1 \leq i_{1}<\ldots<i_{k} \leq n}\left((\varepsilon-1) /\left(\varepsilon^{\eta^{\prime \prime}}-1\right)\right)^{1 / C_{n}^{k}}\right), \eta^{\prime \prime}=1-\log _{\varepsilon}\left(1+(\varepsilon-1) / \prod_{h=1}^{k}\left((\varepsilon-1) /\left(\varepsilon^{1-\eta^{\prime}}-1\right)\right)\right), \\
& \eta^{\prime}=\log _{\varepsilon}\left(1+\left(\varepsilon^{\eta_{i_{h}}}-1\right)^{n \omega_{i_{h}}} /(\varepsilon-1)^{n \omega_{i_{h}}-1}\right), \\
& v^{\prime \prime \prime}=\log _{\varepsilon}\left(1+(\varepsilon-1) / \prod_{1 \leq i_{1}<\ldots<i_{k} \leq n}\left((\varepsilon-1) /\left(\varepsilon^{v^{\prime \prime}}-1\right)\right)^{1 / c_{n}^{k}}\right), v^{\prime \prime}=1-\log _{\varepsilon}\left(1+(\varepsilon-1) / \prod_{h=1}^{k}\left((\varepsilon-1) /\left(\varepsilon^{1-v^{\prime}}-1\right)\right)\right), \\
& v^{\prime}=\log _{\varepsilon}\left(1+\left(\varepsilon^{v_{l_{h}}}-1\right)^{n \omega_{l_{h}}} /(\varepsilon-1)^{n \omega_{i_{h}}-1}\right)
\end{aligned}
$$

\subsection{PFAPWMSM Operator}

To capture the relative importance of the aggregated PFNs, a picture fuzzy Archimedean power weighted MSM (PFAPWMSM) operator is presented. This operator can be formally defined as:

Definition 10. Suppose $w_{1}, w_{2}, \ldots, w_{n}\left(0 \leq w_{1}, w_{2}, \ldots, w_{n} \leq 1\right.$ and $\left.w_{1}+w_{2}+\ldots+w_{n}=1\right)$ are respectively the weights of $\alpha_{1}, \alpha_{2}$, $\ldots, \alpha_{n}$ that respectively denote the relative importance of $\alpha_{1}, \alpha_{2}, \ldots, \alpha_{n}$. Then on the basis of Def. 9 ,

$$
\operatorname{PFAPWMSM}^{(k)}\left(\alpha_{1}, \alpha_{2}, \ldots, \alpha_{n}\right)=\left(\frac{1}{C_{n}^{k}} \underset{1 \leq i_{1}<\ldots<i_{k} \leq n}{\bigotimes} \bigotimes_{h=1}^{k}\left(\frac{n w_{i_{h}}\left(1+T\left(\alpha_{i_{h}}\right)\right)}{\sum_{j=1}^{n}\left(w_{j}\left(1+T\left(\alpha_{j}\right)\right)\right)} \alpha_{i_{h}}\right)\right)^{1 / k}
$$

is called the PFAPWMSM operator.

The general expression of the PFAPWMSM is constructed in the following theorem:

Theorem 5. Suppose $\alpha_{1}, \alpha_{2}, \ldots, \alpha_{n}\left(\alpha_{i}=\left\langle\mu_{i}, \eta_{i}, v_{i}\right\rangle, i=1,2, \ldots, n\right)$ are $n$ PFNs. Then

$$
\operatorname{PFAPWMSM}^{(k)}\left(\alpha_{1}, \alpha_{2}, \ldots, \alpha_{n}\right)=\langle\mu, \eta, v\rangle
$$

where

$$
\begin{aligned}
& \left.\mu=\varphi^{-1}\left(\frac{1}{k} \varphi\left(\psi^{-1}\left(\frac{1}{C_{n}^{k}} \sum_{1 \leq i_{i}<\ldots<i_{k} \leq n} \psi\left(\varphi^{-1}\left(\sum_{h=1}^{k} \varphi\left(\psi^{-1}\left(\left(n w_{i_{h}} \omega_{i_{h}}\right) / \sum_{t=1}^{n}\left(w_{t} \omega_{t}\right)\right) \psi\left(\mu_{i_{h}}\right)\right)\right)\right)\right)\right)\right)\right) \\
& \left.\eta=\psi^{-1}\left(\frac{1}{k} \psi\left(\varphi^{-1}\left(\frac{1}{C_{n}^{k}} \sum_{1 \leq i_{1}<\ldots<i_{k} \leq n} \varphi\left(\psi^{-1}\left(\sum_{h=1}^{k} \psi\left(\varphi^{-1}\left(\left(n w_{i_{h}} \omega_{i_{h}}\right) / \sum_{t=1}^{n}\left(w_{t} \omega_{t}\right)\right) \varphi\left(\eta_{i_{h}}\right)\right)\right)\right)\right)\right)\right)\right) \\
& \left.v=\psi^{-1}\left(\frac{1}{k} \psi\left(\varphi^{-1}\left(\frac{1}{C_{n}^{k}} \sum_{1 \leq i_{1}<\ldots<i_{k} \leq n} \varphi\left(\psi^{-1}\left(\sum_{h=1}^{k} \psi\left(\varphi^{-1}\left(\left(n w_{i_{h}} \omega_{i_{h}}\right) / \sum_{t=1}^{n}\left(w_{t} \omega_{t}\right)\right) \varphi\left(v_{i_{h}}\right)\right)\right)\right)\right)\right)\right)\right) \\
& \omega_{i_{h}}=\left(1+T\left(\alpha_{i_{h}}\right)\right) / \sum_{j=1}^{n}\left(1+T\left(\alpha_{j}\right)\right) \\
& \omega_{t}=\left(1+T\left(\alpha_{t}\right)\right) / \sum_{j=1}^{n}\left(1+T\left(\alpha_{j}\right)\right)
\end{aligned}
$$

and $\operatorname{PFAPWMSM}^{(k)}\left(\alpha_{1}, \alpha_{2}, \ldots, \alpha_{n}\right)$ is still a PFN.

This theorem can be proved like proof Theorem 1 in App. A. The properties of the PFAPWMSM operator are stated in the following theorems:

Theorem 6 (Commutativity). Suppose $\alpha_{1}, \alpha_{2}, \ldots, \alpha_{n}\left(\alpha_{i}=\left\langle\mu_{i}, \eta_{i}, v_{i}\right\rangle, i=1,2, \ldots, n\right)$ are $n$ PFNs. If $\left(\beta_{1}, \beta_{2}, \ldots, \beta_{n}\right)$ is any permutation of $\left(\alpha_{1}, \alpha_{2}, \ldots, \alpha_{n}\right)$, then $\operatorname{PFAPWMSM}^{(k)}\left(\beta_{1}, \beta_{2}, \ldots, \beta_{n}\right)=\operatorname{PFAPWMSM}^{(k)}\left(\alpha_{1}, \alpha_{2}, \ldots, \alpha_{n}\right)$.

Theorem 7 (Boundedness). Suppose $\alpha_{1}, \alpha_{2}, \ldots, \alpha_{n}\left(\alpha_{i}=\left\langle\mu_{i}, \eta_{i}, v_{i}\right\rangle, i=1,2, \ldots, n\right)$ are $n$ PFNs, $\alpha^{+}=\left\langle\max \left(\mu_{i}\right), \min \left(\eta_{i}\right)\right.$, $\min \left(v_{i}\right)>$, and $\alpha^{-}=\left\langle\min \left(\mu_{i}\right), \max \left(\eta_{i}\right), \max \left(v_{i}\right)\right\rangle$. Then $\alpha^{-} \leq P F A P W M S M^{(k)}\left(\alpha_{1}, \alpha_{2}, \ldots, \alpha_{\xi}\right) \leq \alpha^{+}$.

These theorems can be respectively proved like proof of Theorem 3 in App. C and proof of Theorem 4 in App. D. Some special cases of the PFAPWMSM operator are as follows:

(1) If $k=1$, the PFAPWMSM operator will become 


$$
\begin{aligned}
\left\langle\psi^{-1}\left(\frac{1}{n} \sum_{i=1}^{n}\left(\left(\left(n w_{i} \omega_{i}\right) / \sum_{t=1}^{n}\left(w_{t} \omega_{t}\right)\right) \psi\left(\mu_{i}\right)\right)\right), \varphi^{-1}\left(\frac{1}{n} \sum_{i=1}^{n}\left(\left(\left(n w_{i} \omega_{i}\right) / \sum_{t=1}^{n}\left(w_{t} \omega_{t}\right)\right) \varphi\left(\eta_{i}\right)\right)\right),\right. \\
\left.\varphi^{-1}\left(\frac{1}{n} \sum_{i=1}^{n}\left(\left(\left(n w_{i} \omega_{i}\right) / \sum_{t=1}^{n}\left(w_{t} \omega_{t}\right)\right) \varphi\left(v_{i}\right)\right)\right)\right\rangle=\operatorname{PFAPWAA}\left(\alpha_{1}, \alpha_{2}, \ldots, \alpha_{n}\right)
\end{aligned}
$$

which is a picture fuzzy Archimedean power weighted AA (PFAPWAA) operator.

(2) If $k=2$, the PFAPWMSM operator will become

$$
\begin{gathered}
\left\langle\varphi^{-1}\left(\frac{1}{2} \varphi\left(\psi^{-1}\left(\frac{1}{n(n-1)} \sum_{\substack{i_{1}, i_{2}=1 \\
i_{2} \neq i_{1}}}^{n} \psi\left(\varphi^{-1}\left(\varphi\left(\psi^{-1}\left(\frac{n w_{i_{1}} \omega_{i_{1}}}{\sum_{t=1}^{n}\left(w_{t} \omega_{t}\right)} \psi\left(\mu_{i_{1}}\right)\right)\right)+\varphi\left(\psi^{-1}\left(\frac{n w_{i_{2}} \omega_{i_{2}}}{\sum_{t=1}^{n}\left(w_{t} \omega_{t}\right)} \psi\left(\mu_{i_{2}}\right)\right)\right)\right)\right)\right)\right)\right)\right. \\
\left.\psi^{-1}\left(\frac{1}{2} \psi\left(\varphi^{-1}\left(\frac{1}{n(n-1)} \sum_{\substack{i_{1}, i_{2}=1 \\
i_{2} \neq i_{1}}}^{n} \varphi\left(\psi^{-1}\left(\psi\left(\varphi^{-1}\left(\frac{n w_{i_{1}} \omega_{i_{1}}}{\sum_{t=1}^{n}\left(w_{t} \omega_{t}\right)} \varphi\left(\eta_{i_{1}}\right)\right)\right)+\psi\left(\varphi^{-1}\left(\frac{n w_{i_{2}} \omega_{i_{2}}}{\sum_{t=1}^{n}\left(w_{t} \omega_{t}\right)} \varphi\left(\eta_{i_{2}}\right)\right)\right)\right)\right)\right)\right)\right)\right) \\
\psi^{-1}\left(\frac{1}{2} \psi\left(\varphi^{-1}\left(\frac{1}{n(n-1)} \sum_{\substack{i_{1}, i_{2}=1 \\
i_{2} \neq i_{1}}}^{n} \varphi\left(\psi^{-1}\left(\psi\left(\varphi^{-1}\left(\frac{n w_{i_{1}} \omega_{i_{1}}}{\sum_{t=1}^{n}\left(w_{t} \omega_{t}\right)} \varphi\left(v_{i_{1}}\right)\right)\right)+\psi\left(\varphi^{-1}\right)\right)\right)\right)\right)\right) \\
\left.\left.\left.\left.\left.\left.\frac{n w_{i_{2}} \omega_{i_{2}}}{\sum_{t=1}^{n}\left(w_{t} \omega_{t}\right)} \varphi\left(v_{i_{2}}\right)\right)\right)\right)\right)\right)\right)
\end{gathered}
$$

$\operatorname{PFAPWBM}^{(1,1)}\left(\alpha_{1}, \alpha_{2}, \ldots, \alpha_{n}\right)$

which is a picture fuzzy Archimedean power weighted BM (PFAPWBM) operator.

(3) If $k=n$, the PFAPWMSM operator will become

$$
\begin{aligned}
& \left\langle\varphi^{-1}\left(\frac{1}{n} \sum_{i=1}^{n} \varphi\left(\psi^{-1}\left(\left(\left(n w_{i} \omega_{i}\right) / \sum_{t=1}^{n}\left(w_{t} \omega_{t}\right)\right) \psi\left(\mu_{i}\right)\right)\right)\right), \psi^{-1}\left(\frac{1}{n} \sum_{i=1}^{n} \psi\left(\varphi^{-1}\left(\left(\left(n w_{i} \omega_{i}\right) / \sum_{t=1}^{n}\left(w_{t} \omega_{t}\right)\right) \varphi\left(\eta_{i}\right)\right)\right)\right),\right. \\
& \left.\psi^{-1}\left(\frac{1}{n} \sum_{i=1}^{n} \psi\left(\varphi^{-1}\left(\left(\left(n w_{i} \omega_{i}\right) / \sum_{t=1}^{n}\left(w_{t} \omega_{t}\right)\right) \varphi\left(v_{i}\right)\right)\right)\right)\right\rangle=\text { PFAPWGA }\left(\alpha_{1}, \alpha_{2}, \ldots, \alpha_{n}\right)
\end{aligned}
$$

which is a picture fuzzy Archimedean power weighted GA (PFAPWGA) operator.

The specific expressions of the PFAPWMSM operator are constructed as follows:

(1) If Algebraic T-norm and T-conorm are applied to Eq. (20), a picture fuzzy Archimedean Algebraic power weighted MSM (PFAAPWMSM) operator can be obtained as:

$$
\begin{aligned}
& \operatorname{PFAAPWMSM}^{(k)}\left(\alpha_{1}, \alpha_{2}, \ldots, \alpha_{n}\right)=\left\langle\left(1-\left(\prod_{1 \leq i_{1}<\ldots<i_{k} \leq n}\left(1-\prod_{h=1}^{k}\left(1-\left(1-\mu_{i_{h}}\right)^{\left(n w_{i_{h}} \omega_{h_{h}}\right)} / \sum_{t=1}^{n}\left(w_{t} \omega_{t}\right)\right)\right)\right)^{1 / C_{n}^{k}}\right)^{1 / k},\right. \\
& 1-\left(1-\left(\prod_{1 \leq i_{1}<\ldots<i_{k} \leq n}\left(1-\prod_{h=1}^{k}\left(1-\eta_{i_{h}}\left(n w_{i_{h}} \omega_{i_{h}}\right) / \sum_{t=1}^{n}\left(w_{t} \omega_{t}\right)\right)\right)\right)^{1 / c_{n}^{k}}\right)^{1 / k}, \\
& \left.1-\left(1-\left(\prod_{1 \leq i_{1}<\ldots<i_{k} \leq n}\left(1-\prod_{h=1}^{k}\left(1-v_{i_{h}}\left(n w_{i_{h}} \omega_{i_{h}}\right) / \sum_{t=1}^{n}\left(w_{t} \omega_{t}\right)\right)\right)\right)^{1 / c_{n}^{k}}\right)^{1 / k}\right\rangle
\end{aligned}
$$

a) If $k=1$, the PFAAPWMSM operator will become 


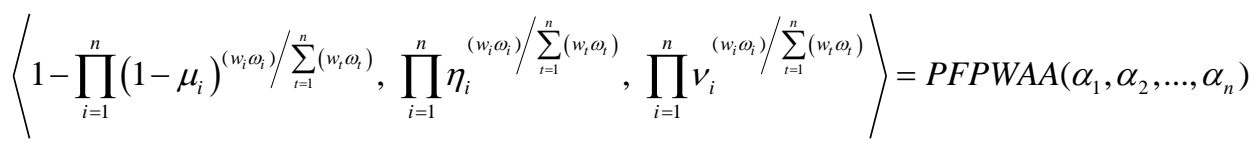

which is a picture fuzzy power weighted AA (PFPWAA) operator.

b) If $k=2$, the PFAAPWMSM operator will become

$$
\begin{aligned}
& \left\langle\left(1-\prod_{\substack{i_{1}, i_{2}=1 \\
i_{2} \neq i_{1}}}^{n}\left(1-\left(1-\left(1-\mu_{i_{1}}\right)^{\left(n w_{i_{1}} \omega_{i_{1}}\right)} / \sum_{t=1}^{n}\left(w_{t} \omega_{t}\right)\right)\left(1-\left(1-\mu_{i_{2}}\right)^{\left(n w_{i_{2}} \omega_{i_{2}}\right)} / \sum_{t=1}^{n}\left(w_{t} \omega_{t}\right)\right)\right)^{\frac{1}{n(n-1)}}\right)^{1 / 2},\right. \\
& \left.1-\left(1-\prod_{\substack{i_{1}, i_{2}=1 \\
i_{2} \neq i_{1}}}^{n}\left(1-\left(1-\eta_{i_{1}}^{\left(n w_{i_{1}} \omega_{i_{1}}\right) / \sum_{t=1}^{n}\left(w_{t} \omega_{t}\right)}\right)\left(1-\eta_{i_{2}}^{\left(n w_{i_{2}} \omega_{i_{2}}\right)}\right) / \sum_{t=1}^{n}\left(w_{t} \omega_{t}\right)\right)\right)^{\frac{1}{n(n-1)}}\right)^{1 / 2},
\end{aligned}
$$

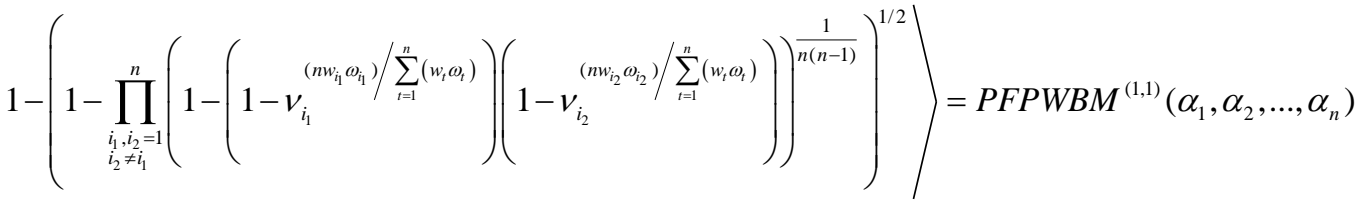

which is a picture fuzzy power weighted BM (PFPWBM) operator.

c) If $k=n$, the PFAAPWMSM operator will become

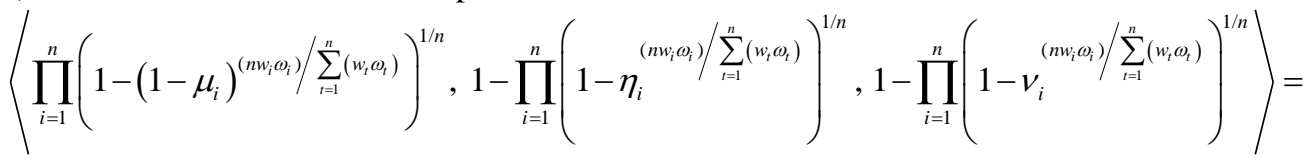

$\operatorname{PFPWGA}\left(\alpha_{1}, \alpha_{2}, \ldots, \alpha_{n}\right)$

which is a picture fuzzy power weighted GA (PFPWGA) operator.

(2) If Einstein T-norm and T-conorm are applied to Eq. (20), a picture fuzzy Archimedean Einstein power weighted MSM (PFAEPWMSM) operator can be obtained as:

$$
\begin{aligned}
& \operatorname{PFAEPWMSM}^{(k)}\left(\alpha_{1}, \alpha_{2}, \ldots, \alpha_{n}\right)=\left\langle\left(2\left(\mu^{\prime \prime}-1\right)^{1 / k}\right) /\left(\left(\mu^{\prime \prime}+3\right)^{1 / k}+\left(\mu^{\prime \prime}-1\right)^{1 / k}\right),\right. \\
& \left.\left(\left(\eta^{\prime \prime}+3\right)^{1 / k}-\left(\eta^{\prime \prime}-1\right)^{1 / k}\right) /\left(\left(\eta^{\prime \prime}+3\right)^{1 / k}+\left(\eta^{\prime \prime}-1\right)^{1 / k}\right),\left(\left(v^{\prime \prime}+3\right)^{1 / k}-\left(v^{\prime \prime}-1\right)^{1 / k}\right) /\left(\left(v^{\prime \prime}+3\right)^{1 / k}+\left(v^{\prime \prime}-1\right)^{1 / k}\right)\right\rangle
\end{aligned}
$$

where

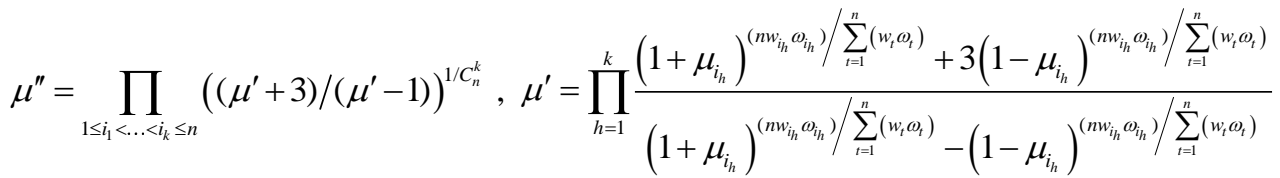

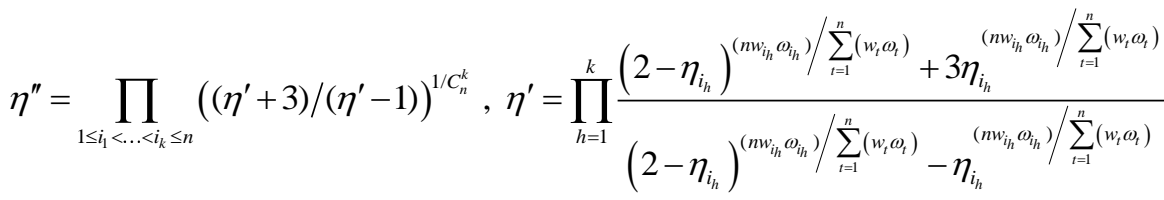

$$
\begin{aligned}
& v^{\prime \prime}=\prod_{1 \leq i_{1}<\ldots<i_{k} \leq n}\left(\left(v^{\prime}+3\right) /\left(v^{\prime}-1\right)\right)^{1 / C_{n}^{k}}, v^{\prime}=\prod_{h=1}^{k} \frac{\left(2-v_{i_{h}}\right)^{\left(n w_{i_{h}} \omega_{i_{h}}\right)} / \sum_{t=1}^{n}\left(w_{t} \omega_{t}\right)}{\left.\left(2-v_{i_{h}}\right)^{\left(n w_{i_{h}} \omega_{i_{h}}\right)}+\sum_{t=1}^{n}\left(w_{t} \omega_{t}\right)-v_{i_{h}}\left(n w_{i_{h}} w_{i_{h}} \omega_{i_{h}}\right) / \sum_{t=1}^{n}\right) / \sum_{t=1}^{n}\left(w_{t} \omega_{t}\right)}
\end{aligned}
$$

(3) If Hamacher T-norm and T-conorm are applied to Eq. (20), a picture fuzzy Archimedean Hamacher power weighted MSM (PFAHPWMSM) operator can be obtained as: 


$$
\begin{aligned}
\operatorname{PFAHPWMSM}^{(k)}\left(\alpha_{1}, \alpha_{2}, \ldots, \alpha_{n}\right)=\langle & \left(\lambda\left(\mu^{\prime \prime}-1\right)^{1 / k}\right) /\left(\left(\mu^{\prime \prime}+\lambda^{2}-1\right)^{1 / k}+(\lambda-1)\left(\mu^{\prime \prime}-1\right)^{1 / k}\right), \\
& \left(\left(\eta^{\prime \prime}+\lambda^{2}-1\right)^{1 / k}-\left(\eta^{\prime \prime}-1\right)^{1 / k}\right) /\left(\left(\eta^{\prime \prime}+\lambda^{2}-1\right)^{1 / k}+(\lambda-1)\left(\eta^{\prime \prime}-1\right)^{1 / k}\right), \\
& \left.\left(\left(v^{\prime \prime}+\lambda^{2}-1\right)^{1 / k}-\left(v^{\prime \prime}-1\right)^{1 / k}\right) /\left(\left(v^{\prime \prime}+\lambda^{2}-1\right)^{1 / k}+(\lambda-1)\left(v^{\prime \prime}-1\right)^{1 / k}\right)\right\rangle
\end{aligned}
$$

where

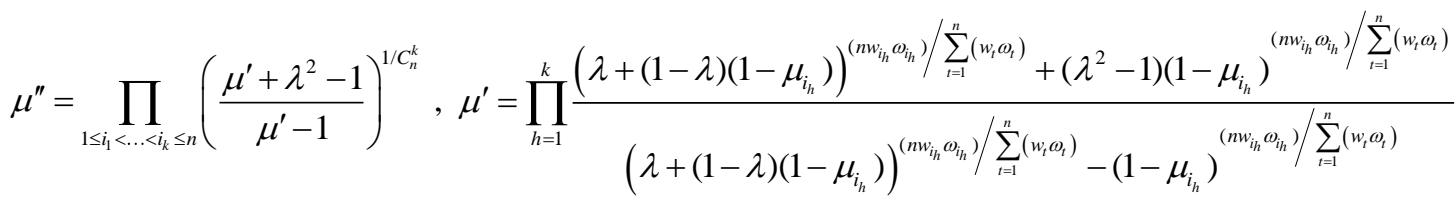

$$
\begin{aligned}
& \eta^{\prime \prime}=\prod_{1 \leq i_{1}<\ldots<i_{k} \leq n}\left(\frac{\eta^{\prime}+\lambda^{2}-1}{\eta^{\prime}-1}\right)^{1 / c_{n}^{k}}, \eta^{\prime}=\prod_{h=1}^{k} \frac{\left(\lambda+(1-\lambda) \eta_{i_{h}}\right)^{\left(n w_{i h} \omega_{i_{h}}\right)} / \sum_{t=1}^{n}\left(w_{t} \omega_{t}\right)+\left(\lambda^{2}-1\right) \eta_{i_{h}}\left(n w_{i_{h}} \omega_{i_{h}}\right) / \sum_{t=1}^{n}\left(w_{t} \omega_{t}\right)}{\left(\lambda+(1-\lambda) \eta_{i_{h}}\right)^{\left(n w_{i_{h}} \omega_{i_{h}}\right)} / \sum_{t=1}^{n}\left(w_{i} \omega_{t}\right)-\eta_{i_{h}}\left(n w_{i_{h}} \omega_{i_{h}}\right) / \sum_{t=1}^{n}\left(w_{i} \omega_{t}\right)}
\end{aligned}
$$

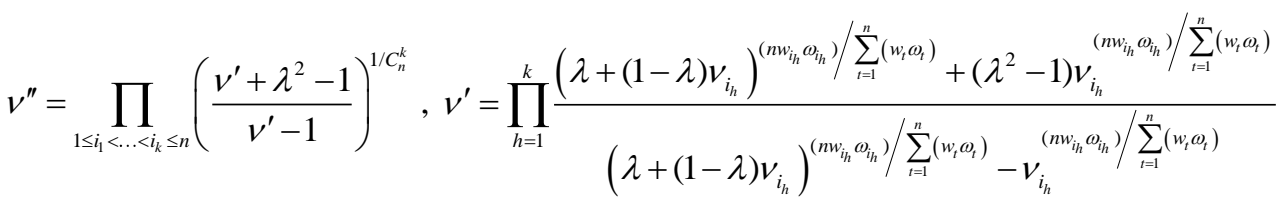

(4) If Frank T-norm and T-conorm are applied to Eq. (20), a picture fuzzy Archimedean Frank power weighted MSM (PFAFPWMSM) operator can be obtained as:

$$
\begin{array}{r}
\operatorname{PFAFPWMSM}^{(k)}\left(\alpha_{1}, \alpha_{2}, \ldots, \alpha_{n}\right)=\left\langle\log _{\varepsilon}\left(1+\left(\varepsilon^{\mu^{\prime \prime \prime}}-1\right)^{1 / k} /(\varepsilon-1)^{1 / k-1}\right),\right. \\
1-\log _{\varepsilon}\left(1+\left(\varepsilon^{1-\eta^{\prime \prime \prime}}-1\right)^{1 / k} /(\varepsilon-1)^{1 / k-1}\right), \\
\left.1-\log _{\varepsilon}\left(1+\left(\varepsilon^{1-\nu^{\prime \prime \prime}}-1\right)^{1 / k} /(\varepsilon-1)^{1 / k-1}\right)\right\rangle
\end{array}
$$

where

$$
\begin{aligned}
& \mu^{\prime \prime \prime}=1-\log _{\varepsilon}\left(1+(\varepsilon-1) / \prod_{1 \leq i_{1}<\ldots<i_{k} \leq n}\left((\varepsilon-1) /\left(\varepsilon^{1-\mu^{\prime \prime}}-1\right)\right)^{1 / C_{n}^{k}}\right), \mu^{\prime \prime}=\log _{\varepsilon}\left(1+(\varepsilon-1) / \prod_{h=1}^{k}\left((\varepsilon-1) /\left(\varepsilon^{\mu^{\prime}}-1\right)\right)\right), \\
& \mu^{\prime}=1-\log _{\varepsilon}\left(1+\left(\left(\varepsilon^{1-\mu_{i_{h}}}-1\right)^{\left(n w_{i_{h}} \omega_{i_{h}}\right) / \sum_{t=1}^{n}\left(w_{t} \omega_{t}\right)} /(\varepsilon-1)^{\left(n w_{h_{h}} \omega_{i_{h}}\right)} / \sum_{t=1}^{n}\left(w_{t} \omega_{t}\right)-1\right)\right), \\
& \eta^{\prime \prime \prime}=\log _{\varepsilon}\left(1+(\varepsilon-1) / \prod_{1 \leq i_{1}<\ldots<i_{k} \leq n}\left((\varepsilon-1) /\left(\varepsilon^{\eta^{\prime \prime}}-1\right)\right)^{1 / C_{n}^{k}}\right), \eta^{\prime \prime}=1-\log _{\varepsilon}\left(1+(\varepsilon-1) / \prod_{h=1}^{k}\left((\varepsilon-1) /\left(\varepsilon^{1-\eta^{\prime}}-1\right)\right)\right), \\
& \eta^{\prime}=\log _{\varepsilon}\left(1+\left(\varepsilon^{\eta_{i_{h}}}-1\right)^{\left(n w_{i_{h}} \omega_{i h}\right)} / \sum_{t=1}^{n}\left(w_{t} \omega_{t}\right) /(\varepsilon-1)^{\left(n w_{i_{h}} \omega_{i_{h}}\right)} / \sum_{t=1}^{n}\left(w_{t} \omega_{t}\right)-1\right), \\
& v^{\prime \prime \prime}=\log _{\varepsilon}\left(1+(\varepsilon-1) / \prod_{1 \leq i_{1}<\ldots<i_{k} \leq n}\left((\varepsilon-1) /\left(\varepsilon^{v^{\prime \prime}}-1\right)\right)^{1 / C_{n}^{k}}\right), v^{\prime \prime}=1-\log _{\varepsilon}\left(1+(\varepsilon-1) / \prod_{h=1}^{k}\left((\varepsilon-1) /\left(\varepsilon^{1-v^{\prime}}-1\right)\right)\right), \\
& v^{\prime}=\log _{\varepsilon}\left(1+\left(\varepsilon^{v_{i_{h}}}-1\right)^{\left(n w_{i h} \omega_{i_{h}}\right)} / \sum_{t=1}^{n}\left(w_{t} \omega_{t}\right) /(\varepsilon-1)^{\left(n w_{i_{h}} \omega_{i h}\right)} / \sum_{t=1}^{n}\left(w_{t} \omega_{t}\right)-1\right)
\end{aligned}
$$

\section{MADM Method}

In an MADM problem based on PFNs, decision makers need to select the most appropriate option from a certain number of options. The selection criterion is usually based on a certain number of attributes, whose relative important is measured by weights. The values of the attributes of each option are given by PFNs. The basic components of an MADM problem based on PFNs include a set of options $\boldsymbol{O}=\left\{O_{1}, O_{2}, \ldots, O_{m}\right\}$, a set of attributes $\boldsymbol{A}=\left\{A_{1}, A_{2}, \ldots, A_{n}\right\}$, a vector of weights of attributes $\boldsymbol{w}=\left[w_{1}, w_{2}, \ldots, w_{n}\right]\left(0 \leq w_{1}, w_{2}, \ldots, w_{n} \leq 1, w_{1}+w_{2}+\ldots+w_{n}=1\right)$, and a picture fuzzy decision matrix $\boldsymbol{M}=\left[\alpha_{i, j}\right]_{m \times n}(i=1$, $2, \ldots, m ; j=1,2, \ldots, n ; \alpha_{i, j}=\left\langle\mu_{i, j}, \eta_{i, j}, v_{i, j}\right\rangle$ is a PFN that is the evaluation value of $A_{j}$ of $\left.O_{i}\right)$. The objective of the MADM problem is to determine the best option from the options in $\boldsymbol{O}$ on the basis of $\boldsymbol{M}$ and $\boldsymbol{w}$. This objective can be achieved through the 
the following steps:

Step 1: Normalise the picture fuzzy decision matrix $\boldsymbol{M}$. In general, an MADM problem may contain benefit and cost attributes, which have opposite influences on the aggregation result. To unify the influences, a complement rule is usually applied to normalise the PFNs expressing the values of cost attributes. Using this rule, the picture fuzzy decision matrix $\boldsymbol{M}=$ $\left[\alpha_{i, j}\right]_{m \times n}$ is normalised according to the following equation:

$$
\boldsymbol{M}_{\mathbf{N}}=\left[\alpha_{i, j}\right]_{m \times n}=\left\{\begin{array}{l}
{\left[\left\langle\mu_{i, j}, \eta_{i, j}, v_{i, j}\right\rangle\right]_{m \times n}, \text { if } A_{j} \text { is a benefit attribute }} \\
{\left[\left\langle v_{i, j}, \eta_{i, j}, \mu_{i, j}\right\rangle\right]_{m \times n}, \text { if } A_{j} \text { is a cost attribute }}
\end{array}\right.
$$

Step 2: Compute the weights of $\alpha_{i, j}$. Based on Def.s 9 and 10 and Theorem 5, the weights of $\alpha_{i, j}$ are calculated using

$$
\varpi_{i, j}=\left(w_{j} \omega_{j}\right) / \sum_{t=1}^{n}\left(w_{t} \omega_{t}\right)=\left(w_{j}\left(1+\sum_{p=1, p \neq j}^{n}\left(1-d\left(\alpha_{i, j}, \alpha_{i, p}\right)\right)\right)\right) / \sum_{t=1}^{n}\left(w_{t}\left(1+\sum_{q=1, q \neq t}^{n}\left(1-d\left(\alpha_{i, t}, \alpha_{i, q}\right)\right)\right)\right)
$$

where $d\left(\alpha_{i, j}, \alpha_{i, p}\right)$ and $d\left(\alpha_{i, t}, \alpha_{i, q}\right)$ are respectively the normalised Hamming distances of $\alpha_{i, j}$ and $\alpha_{i, p}$ and $\alpha_{\mathrm{i}, t}$ and $\alpha_{i, q}$. They can be calculated according to Def. 5 .

Step 3: Compute the summary values of $\alpha_{i, j}$. The summary values of $\alpha_{i, j}$ are calculated via

$$
\alpha_{i}=\left\langle\mu_{i}, \eta_{i}, v_{i}\right\rangle=\operatorname{PFAPWMSM}^{(k)}\left(\alpha_{i, 1}, \alpha_{i, 2}, \ldots, \alpha_{i, n}\right)
$$

where PFAPWMSM is a specific PFAPWMSM operator, such as the specific operators in Eq.s (24), (28), (29), and (30).

Step 4: Compute the score and accuracy values of $\alpha_{i}$. According to Def.s 2 and 3, the score and accuracy values of $\alpha_{i}$ are respectively calculated.

Step 5: Rank $O_{i}$. According to Def. 4 and the score and accuracy values of $\alpha_{i}$, all $O_{i}$ are ranked.

Step 6: Determine the best option. The best option is generally the option ranked first.

\section{Example, Experiments, and Comparisons}

\subsection{Example}

An MADM example about selection of the best emerging technology enterprise from five viable enterprises (Jana et al. 2019) is introduced to illustrate the proposed MADM method. The five viable enterprises are $E_{1}, E_{2}, E_{3}, E_{4}$, and $E_{5}$. There are four attributes for decision making, which are the technical advancement $\left(A_{1}\right)$, the potential market $\left(A_{2}\right)$, the industrialisation framework, human resources, and financial investments $\left(A_{3}\right)$, and the employment formation and progress of science and technology $\left(A_{4}\right)$. The weights of these attributes is given by $\boldsymbol{w}=[0.2,0.1,0.3,0.4]$. To provide enough freedom in the evaluation of attribute values, PFNs were used. The evaluation results form a picture fuzzy decision matrix $\boldsymbol{M}=\left[\alpha_{i, j}\right]_{5 \times 4}$, whose elements are listed in Table 2.

Table 2. The Elements of the Picture Fuzzy Decision Matrix M

\begin{tabular}{lllll}
\hline \hline & $A_{1}$ & $A_{2}$ & $A_{3}$ & $A_{4}$ \\
\hline$E_{1}$ & $\langle 0.56,0.34,0.10\rangle$ & $\langle 0.90,0.07,0.03\rangle$ & $\langle 0.40,0.33,0.19\rangle$ & $<0.09,0.79,0.03\rangle$ \\
$E_{2}$ & $\langle 0.70,0.10,0.09\rangle$ & $\langle 0.10,0.66,0.20\rangle$ & $\langle 0.06,0.81,0.12\rangle$ & $\langle 0.72,0.14,0.09\rangle$ \\
$E_{3}$ & $\langle 0.88,0.09,0.03\rangle$ & $\langle 0.08,0.10,0.06\rangle$ & $\langle 0.05,0.83,0.09\rangle$ & $\langle 0.65,0.25,0.07\rangle$ \\
$E_{4}$ & $\langle 0.80,0.07,0.04\rangle$ & $\langle 0.70,0.15,0.11\rangle$ & $\langle 0.03,0.88,0.05\rangle$ & $\langle 0.07,0.82,0.05\rangle$ \\
$E_{5}$ & $\langle 0.85,0.06,0.03\rangle$ & $\langle 0.64,0.07,0.22\rangle$ & $\langle 0.06,0.88,0.05\rangle$ & $\langle 0.13,0.77,0.09\rangle$ \\
\hline \hline
\end{tabular}

According to the conditions above and the proposed MADM method, selection of the best emerging technology enterprise can be carried out as follows:

Step 1: Normalise the picture fuzzy decision matrix $\boldsymbol{M}$. Since all of the four attributes are benefit attributes, the normalised picture fuzzy decision matrix $\boldsymbol{M}_{\mathbf{N}}=\boldsymbol{M}=\left[\alpha_{i, j}\right]_{5 \times 4}$.

Step 2: Compute the weights of $\alpha_{i, j}$. On the basis of Eq. (32), the weights of $\alpha_{i, j}$ are calculated as:

$$
\left[\varpi_{i, j}\right]_{5 \times 4}=\left[\begin{array}{llll}
0.2296 & 0.0921 & 0.3342 & 0.3441 \\
0.2031 & 0.1000 & 0.2874 & 0.4095 \\
0.1979 & 0.1094 & 0.2549 & 0.4378 \\
0.1905 & 0.1006 & 0.2968 & 0.4121 \\
0.1894 & 0.0994 & 0.2917 & 0.4195
\end{array}\right]
$$


Step 3: Compute the summary values of $\alpha_{i, j}$. The summary values of $\alpha_{i, j}$ can be calculated via Eq. (33). Here the specific operator in Eq. (29) $(\lambda=3$ and $k=3)$ is leveraged in Eq. (33). The calculated summary values are as follows:

$\alpha_{1}=\langle 0.3965,0.4461,0.1450\rangle, \alpha_{2}=\langle 0.3205,0.4746,0.2026\rangle, \alpha_{3}=\langle 0.3366,0.3767,0.1287\rangle$,

$\alpha_{4}=\langle 0.2318,0.5861,0.1435\rangle, \alpha_{5}=\langle 0.2895,0.5283,0.1641\rangle$

Step 4: Compute the score and accuracy values of $\alpha_{i}$. According to Def.s 2 and 3, the score and accuracy values of $\alpha_{i}$ are calculated as:

$S\left(\alpha_{1}\right)=0.6258, S\left(\alpha_{2}\right)=0.5589, S\left(\alpha_{3}\right)=0.6039, S\left(\alpha_{4}\right)=0.5441, S\left(\alpha_{5}\right)=0.5627$

$A\left(\alpha_{1}\right)=0.9876, A\left(\alpha_{2}\right)=0.9978, A\left(\alpha_{3}\right)=0.8421, A\left(\alpha_{4}\right)=0.9614, A\left(\alpha_{5}\right)=0.9819$

Step 5: Rank $E_{i}$. On the basis of Def. 4, all $E_{i}$ are ranked as:

$E_{1}>E_{3}>E_{5}>E_{2}>E_{4}$

Step 6: Determine the best enterprise. The best enterprise is $E_{1}$ as it is ranked first.

\subsection{Experiments}

To study the impact of different ATT operations and different argument values on the aggregation result, the following experiments were conducted:

(1) Experiment 1. This experiment aims to study the impact of different ATT operations on the aggregation result. In it, the specific AOs using different ATT operations in Eq.s (24), (28), (29), and (30) with $k=3$ and $\lambda=\varepsilon=3$ were respectively used in the numerical example. The experiment results, as depicted in Fig. 1, are the score values of $\alpha_{i}$ and the rankings of $E_{i}$. As can been seen from Fig. 1, the generated rankings of $E_{i}$ under different ATT operations just have difference at the fourth and fifth places, which indicates that different ATT operations have no obvious impact on the aggregation result for the numerical example. Please note that this does not mean that an arbitrary ATT operation can be used in all MADM problems based on PFNs. Whether an ATT operation is suitable for a specific problem should be judged according to the characteristics of the problem.

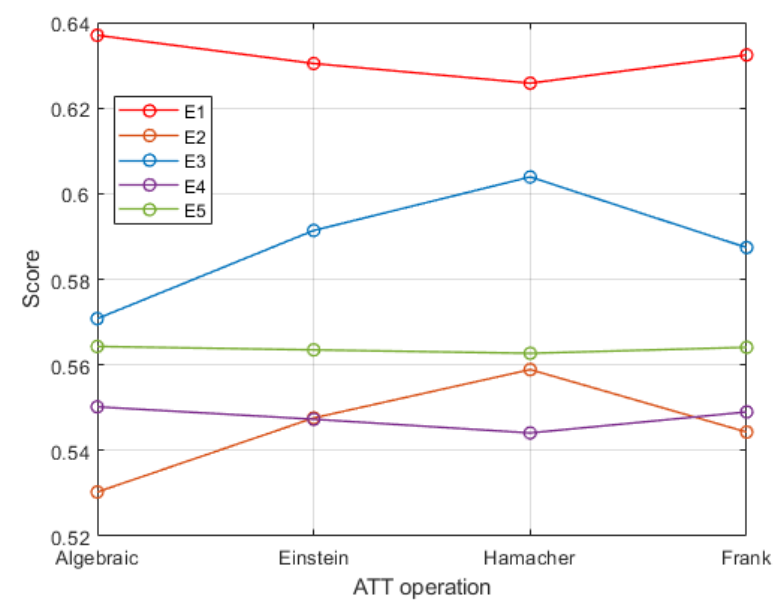

Figure 1. The result of Experiment 1.

(2) Experiment 2. This experiment aims to study the impact of different values of $k$ on the aggregation result. In it, the presented specific AOs in Eq.s (24), (28), (29), and (30) with $k=1,2,3,4$ were respectively used in the numerical example. The experiment results, as listed in Table 3, are the score values of $\alpha_{i}$ and the rankings of $E_{i}$. From Table 3, the score value of each enterprise become smaller and smaller and the generated rankings and best enterprises are different from $k=1$ to $k=4$ for each AO. This indicates that the argument $k$ in each AO reflects the risk attitude and the risk attitude changes from optimism to pessimism as $k$ changes from 1 to 4 . It is worth nothing that MSM will reduce to AA and all attributes are independent of each other when $k=1$, MSM will reduce to BM and there are interactions between any two attributes when $k=2$, and MSM will reduce to GA and there are interactions among the four attributes when $k=4$. Thus the order of the optimism of AA, BM, $\mathrm{MSM}$, and GA is $\mathrm{AA}>\mathrm{BM}>\mathrm{MSM}>\mathrm{GA}$ from the results of the experiment. In practical MADM problems, the value of $k$ should be assigned on the basis of the interactions of attributes. 
Table 3. The Results of Experiment 2

\begin{tabular}{|c|c|c|c|c|c|c|c|c|c|}
\hline \multirow{3}{*}{$\begin{array}{l}\text { Specific AO } \\
\text { used in Eq. (33) } \\
\text { PFAAPWMSM }\end{array}$} & \multirow{2}{*}{$\begin{array}{l}\text { Value } \\
\text { of } k\end{array}$} & \multirow{2}{*}{$\begin{array}{l}\text { Value } \\
\text { of } \lambda(\varepsilon)\end{array}$} & \multicolumn{5}{|c|}{ The calculated score values of the five enterprises } & \multirow{2}{*}{ The generated ranking } & \multirow{2}{*}{$\begin{array}{l}\text { The best } \\
\text { enterprise }\end{array}$} \\
\hline & & & $S\left(\alpha_{1}\right)$ & $S\left(\alpha_{2}\right)$ & $S\left(\alpha_{3}\right)$ & $S\left(\alpha_{4}\right)$ & $S\left(\alpha_{5}\right)$ & & \\
\hline & $k=1$ & - & 0.6900 & 0.7210 & 0.7660 & 0.6605 & 0.6742 & $E_{3}>E_{2}>E_{1}>E_{5}>E_{4}$ & $E_{3}$ \\
\hline & $k=2$ & & 0.6542 & 0.6126 & 0.6735 & 0.5933 & 0.6040 & $E_{3}>E_{1}>E_{2}>E_{5}>E_{4}$ & $E_{3}$ \\
\hline & $k=3$ & & 0.6370 & 0.5303 & 0.5708 & 0.5502 & 0.5643 & $E_{1}>E_{3}>E_{5}>E_{4}>E_{2}$ & $E_{1}$ \\
\hline & $k=4$ & & 0.6170 & 0.4878 & 0.5343 & 0.5151 & 0.5210 & $E_{1}>E_{3}>E_{5}>E_{4}>E_{2}$ & $E_{1}$ \\
\hline \multirow[t]{4}{*}{ PFAEPWMSM } & $k=1$ & 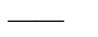 & 0.6752 & 0.7065 & 0.7490 & 0.6352 & 0.6499 & $E_{3}>E_{2}>E_{1}>E_{5}>E_{4}$ & $E_{3}$ \\
\hline & $k=2$ & & 0.6443 & 0.6171 & 0.6736 & 0.5873 & 0.6009 & $E_{3}>E_{1}>E_{2}>E_{5}>E_{4}$ & $E_{3}$ \\
\hline & $k=3$ & & 0.6304 & 0.5476 & 0.5914 & 0.5473 & 0.5635 & $E_{1}>E_{3}>E_{5}>E_{2}>E_{4}$ & $E_{1}$ \\
\hline & $k=4$ & - & 0.6111 & 0.4996 & 0.5441 & 0.5128 & 0.5251 & $E_{1}>E_{3}>E_{5}>E_{4}>E_{2}$ & $E_{1}$ \\
\hline \multirow[t]{4}{*}{ PFAHPWMSM } & $k=1$ & $\lambda=3$ & 0.6673 & 0.6972 & 0.7386 & 0.6205 & 0.6366 & $E_{3}>E_{2}>E_{1} \succ E_{5}>E_{4}$ & $E_{3}$ \\
\hline & $k=2$ & $\lambda=3$ & 0.6385 & 0.6211 & 0.6747 & 0.5821 & 0.5982 & $E_{3}>E_{1}>E_{2}>E_{5}>E_{4}$ & $E_{3}$ \\
\hline & $k=3$ & $\lambda=3$ & 0.6258 & 0.5589 & 0.6039 & 0.5441 & 0.5627 & $E_{1}>E_{3}>E_{5}>E_{2}>E_{4}$ & $E_{1}$ \\
\hline & $k=4$ & $\lambda=3$ & 0.6068 & 0.5078 & 0.5509 & 0.5116 & 0.5279 & $E_{1}>E_{3}>E_{5}>E_{4} \succ E_{2}$ & $E_{1}$ \\
\hline \multirow[t]{4}{*}{ PFAFPWMSM } & $k=1$ & $\varepsilon=3$ & 0.6790 & 0.7110 & 0.7536 & 0.6433 & 0.6568 & $E_{3}>E_{2}>E_{1}>E_{5}>E_{4}$ & $E_{3}$ \\
\hline & $k=2$ & $\varepsilon=3$ & 0.6469 & 0.6172 & 0.6746 & 0.5907 & 0.6027 & $E_{3}>E_{1}>E_{2}>E_{5}>E_{4}$ & $E_{3}$ \\
\hline & $k=3$ & $\varepsilon=3$ & 0.6324 & 0.5443 & 0.5874 & 0.5490 & 0.5641 & $E_{1}>E_{3}>E_{5}>E_{4}>E_{2}$ & $E_{1}$ \\
\hline & $k=4$ & $\varepsilon=3$ & 0.6124 & 0.4971 & 0.5420 & 0.5134 & 0.5238 & $E_{1}>E_{3} \succ E_{5}>E_{4}>E_{2}$ & $E_{1}$ \\
\hline
\end{tabular}

(3) Experiment 3. This experiment aims to study the impact of different values of $\lambda(\varepsilon)$ on the aggregation result. In it, the PFAHPWMSM operator in Eq. (29) with $k=3$ and $0.01 \leq \lambda \leq 20.00$ and the PFAFPWMSM operator in Eq. (30) with $k=3$ and $1.01 \leq \varepsilon \leq 20.00$ were respectively leveraged in the numerical example. The experiment results, as depicted in Fig.s 2 and 3 , are the score values of $\alpha_{i}$ and the rankings of $E_{i}$. From Fig.s 2 and 3, the scores calculated by the two operators decrease or increase and the generated rankings have minor changes as the values of $\lambda$ and $\varepsilon$ gradually increase. This shows that there is no fixed rule for the influence of $\lambda$ and $\varepsilon$ on the aggregation result for the numerical example, although the two arguments can be used as risk attitude factors in other practical MADM problems. In general, an appropriate $\lambda(\varepsilon)$ value (e.g. $\lambda=1,2,3 ; \varepsilon=2,3,4)$ is recommended when the PFAHPWMSM (PFAFPWMSM) operator is used.

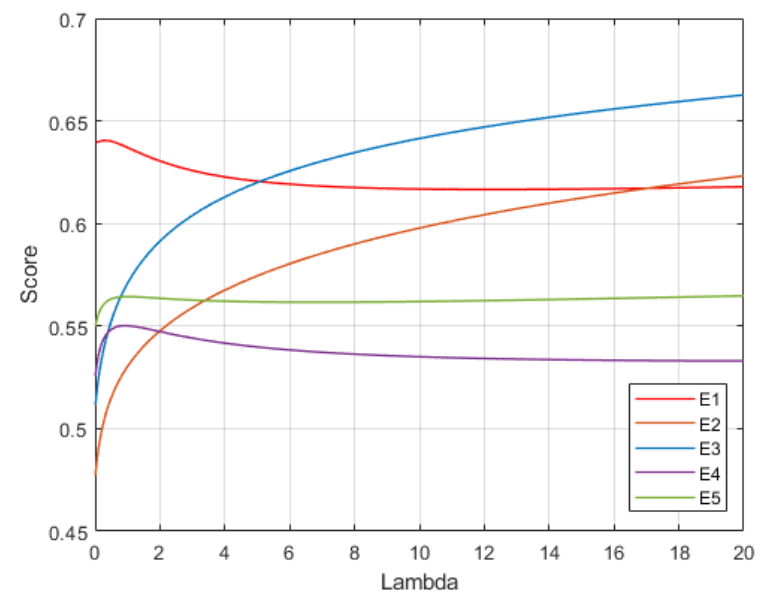

Figure 2. The result of Experiment 3 when PFAHPWMSM is used. 


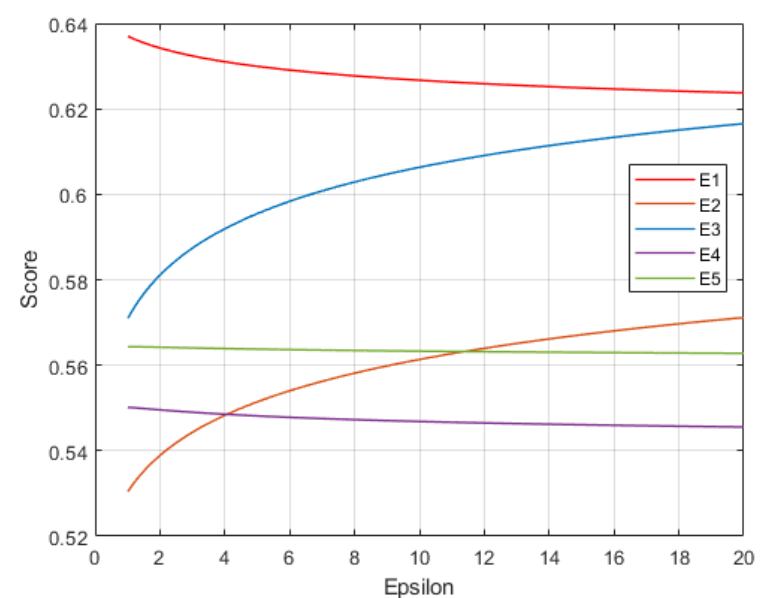

Figure 3. The result of Experiment 3 when PFAFPWMSM is used.

\subsection{Comparisons}

Representative MADM methods based on AOs of PFNs are the methods presented by Wei (2017), Garg (2017b), Wei (2018), Jana et al. (2019), Wei et al. (2018a), Zhang et al. (2018), and Xu et al. (2019). A qualitative comparison and a quantitative comparison between them and the proposed MADM method are respectively carried out to validate the proposed method:

(1) Qualitative comparison. The qualitative comparison was made through comparing the features of the AOs. For the eight methods, the flexibility in the aggregation of attribute values, the generality in the consideration of attribute interactions, and the capability to reduce the negative impact of biased attribute values are used as the comparison characteristics. The comparison results are listed in Table 4:

a) Flexibility in the aggregation of attribute values. The methods of Wei (2017), Wei et al. (2018a), and Xu et al. (2019) realise the aggregation through the operational rules based on Algebraic T-norm and T-conorm, their flexibility is relatively limited. The methods of Wei (2018), Jana et al. (2019), and Zhang et al. (2018) leverage the Hamacher, Dombi, and Dombi Tnorms and T-conorms to carry out their aggregations, respectively. Their flexibility can be seen as moderate because each of the two T-norms and T-conorms can provide a flexible argument for aggregation. The flexibility of the method of Garg (2017b) and the proposed method are satisfying since their aggregations can be realised via the operational rules based on any family of ATTs.

b) Generality in the consideration of attribute interactions. All of the comparison methods can handle the case where all attributes are independent of each other. The methods of Wei et al. (2018a) and Zhang et al. (2018) are applicable for the independence situation and the situation in which there are interactions between any two attributes because of the use of Heronian mean operator in them. The method of Xu et al. (2019) and the proposed method have the most desirable generality in the consideration of attribute interactions because of the use of two all-in-one AOs for capturing relationships, i.e. the Muirhead mean and MSM operators.

c) Capability to reduce the negative impact of biased attribute values. Among all of the eight methods, the method of Wei (2018) and the proposed method have this capability since both of them combine the PA operator.

Table 4. The Results of the Qualitative Comparison

\begin{tabular}{|c|c|c|c|c|c|}
\hline \multirow[b]{2}{*}{ MADM method } & \multirow{2}{*}{$\begin{array}{l}\text { Flexibility in } \\
\text { aggregation of } \\
\text { attribute values }\end{array}$} & \multicolumn{3}{|c|}{ Generality in consideration of interactions } & \multirow{2}{*}{$\begin{array}{l}\text { Capability } \\
\text { to reduce } \\
\text { influence }\end{array}$} \\
\hline & & Independent & Two & Multiple & \\
\hline Wei (2017) & Limited & Yes & No & No & No \\
\hline $\operatorname{Garg}(2017 b)$ & Satisfying & Yes & No & No & No \\
\hline Wei (2018) & Moderate & Yes & No & No & Yes \\
\hline Jana et al. (2019) & Moderate & Yes & No & No & No \\
\hline Wei et al. (2018a) & Limited & Yes & Yes & No & No \\
\hline Zhang et al. (2018) & Moderate & Yes & Yes & No & No \\
\hline Xu et al. (2019) & Limited & Yes & Yes & Yes & No \\
\hline The proposed method & Satisfying & Yes & Yes & Yes & Yes \\
\hline
\end{tabular}

(2) Quantitative comparison. The quantitative comparison was made leveraging the numerical examples in Ref. (Jana et al. 2019) (Example 1), Ref. (Wei 2017) (Example 2), and Ref. (Garg 2017b) (Example 3) as the benchmarks. The WA, Hamacher WA, Hamacher WA, Dombi WA, weighted Heronian mean, Dombi weighted Heronian mean, weighted Muirhead mean, and 
PFAHPWMSM operators were respectively used in the methods in Wei (2017), Garg (2017b), Wei (2018), Jana et al. (2019), Wei et al. (2018a), Zhang et al. (2018), Xu et al. (2019), and the present paper. In addition, the same function for calculating the score values was used in all of these methods for to unify the comparison. The comparison results are listed in Table 5.

Table 5. The Results of the Quantitative Comparison

\begin{tabular}{|c|c|c|c|c|c|c|c|c|c|}
\hline \multirow{2}{*}{ Benchmark } & \multirow{2}{*}{$\begin{array}{l}\text { MADM } \\
\text { method }\end{array}$} & \multirow{2}{*}{$\begin{array}{l}\text { Value of } \\
\text { arguments }\end{array}$} & \multicolumn{5}{|c|}{ The calculated score values of all options } & \multirow{2}{*}{ The generated ranking } & \multirow{2}{*}{$\begin{array}{l}\text { The best } \\
\text { option }\end{array}$} \\
\hline & & & $S\left(\alpha_{1}\right)$ & $S\left(\alpha_{2}\right)$ & $S\left(\alpha_{3}\right)$ & $S\left(\alpha_{4}\right)$ & $S\left(\alpha_{5}\right)$ & & \\
\hline \multirow{5}{*}{ Example 1} & Garg (2017b), Wei (2018) & $\gamma=3$ & 0.6630 & 0.6929 & 0.7286 & 0.6245 & 0.6419 & $O_{3}>O_{2}>O_{1}>O_{5}>O_{4}$ & $\mathrm{O}_{3}$ \\
\hline & Wei et al. (2018a) & $p=1, q=2$ & 0.9147 & 0.8880 & 0.8988 & 0.8933 & 0.8964 & $O_{1}>O_{3}>O_{5}>O_{4}>O_{2}$ & $O_{1}$ \\
\hline & Zhang et al. (2018) & $\lambda=3, p=1, q=2$ & 0.7965 & 0.6834 & 0.7929 & 0.7295 & 0.7627 & $O_{1}>O_{3}>O_{5}>O_{4}>O_{2}$ & $O_{1}$ \\
\hline & Xu et al. (2019) & $P=(1,2,3,0)$ & 0.6485 & 0.5948 & 0.6398 & 0.5934 & 0.6052 & $O_{1}>O_{3}>O_{5}>O_{2}>O_{4}$ & $O_{1}$ \\
\hline & The proposed method & $\lambda=3, k=3$ & 0.6258 & 0.5589 & 0.6039 & 0.5441 & 0.5627 & $O_{1}>O_{3}>O_{5}>O_{2}>O_{4}$ & $O_{1}$ \\
\hline \multirow{5}{*}{ Example 2} & Jana et al. (2019) & $R=3$ & 0.8819 & 0.8022 & 0.9117 & 0.8584 & 0.9038 & $O_{3}>O_{5}>O_{1}>O_{4}>O_{2}$ & $\mathrm{O}_{3}$ \\
\hline & Wei et al. (2018a) & $p=1, q=2$ & 0.9140 & 0.8907 & 0.9040 & 0.9004 & 0.9044 & $O_{1}>O_{5}>O_{3}>O_{4}>O_{2}$ & $O_{1}$ \\
\hline & Zhang et al. (2018) & $\lambda=3, p=1, q=2$ & 0.7929 & 0.6990 & 0.8360 & 0.7639 & 0.8222 & $O_{3}>O_{5}>O_{1}>O_{4}>O_{2}$ & $\mathrm{O}_{3}$ \\
\hline & Xu et al. (2019) & $P=(1,2,3,0)$ & 0.6473 & 0.5920 & 0.6433 & 0.6026 & 0.6168 & $O_{1}>O_{3}>O_{5}>O_{4}>O_{2}$ & $O_{1}$ \\
\hline & The proposed method & $\lambda=3, k=3$ & 0.6293 & 0.5529 & 0.6058 & 0.5525 & 0.5739 & $O_{1}>O_{3}>O_{5}>O_{2}>O_{4}$ & $O_{1}$ \\
\hline \multirow[t]{2}{*}{ Example 3} & Wei (2017) & - & 0.4558 & 0.2868 & 0.4260 & 0.6038 & & $O_{4}>O_{1}>O_{3}>O_{2}$ & $\mathrm{O}_{4}$ \\
\hline & The proposed method & $\lambda=3, k=3$ & 0.3701 & 0.2714 & 0.3907 & 0.5191 & - & $O_{4}>O_{3}>O_{1}>O_{2}$ & $\mathrm{O}_{4}$ \\
\hline
\end{tabular}

From Table 5, the ranking of the proposed method has no significant difference with the rankings of the methods of Jana et al. (2019), Wei et al. (2018a), Zhang et al. (2018), and Xu et al. (2019) for Example 1, with the rankings of the methods of Wei et al. (2018a) and Xu et al. (2019) for Example 2, and with the rankings of all remaining methods for Example 3. This demonstrates that for practical MADM problems based on PFNs, the proposed method is feasible and effective. From Table 4, the method of Xu et al. (2019) and the proposed method are the most similar in characteristics. From Table 5, the rankings of the two methods are exactly identical or have no obvious difference with each other for all examples, which also validates the proposed method. As can also be seen from Table 5, the ranking and best option of the proposed method are fully different with the ranking and best option of the methods of Wei (2017), Garg (2017b), Wei (2018) for Example 1 and of the methods of Wei (2017), Garg (2017b), Wei (2018), Jana et al. (2019), and Zhang et al. (2018) for Example 2. This is because the specific AOs on which these methods are based are different from the specific AO in the proposed method, so do their mathematical properties.

It can be concluded from the results in Table 4 that the proposed MADM method can provide satisfying flexibility in the aggregation of attribute values and the consideration of attribute interactions and concurrently has the capability to reduce the negative impact of biased attribute values on aggregation result. The former characteristic is obvious. But the latter one is not intuitive. To show the difference between a method that has the capability to reduce negative impact and a method that does not have such capability, an additional quantitative comparison was carried out. In this comparison, the weighted Muirhead mean operator in the method of Xu et al. (2019) with $P=(1,1,1,0)$ and the PFAHPWMSM operator in Eq. (29) with $\lambda=1$ and $k=3$ were selected to solve the MADM problem in Example 1. Please note that the only difference of the two operators under such conditions is whether combines the PA operator (i.e. whether has the capability). Suppose the value of attribute $A_{2}$ of enterprise $E_{1}$ is a biased value. The degree of positive membership of this value was constantly reduced based on Table 6 . It can be guessed that this adjustment will have influence on the ranking of $E_{1}$, which could be dropped from the first enterprise to the last one. To confirm this conjecture, Table 6 and Figure 4 list the variation of the places of $E_{1}$ in the rankings generated by the method of Xu et al. (2019) and the proposed method.

Table 6. The Results of the Additional Quantitative Comparison

\begin{tabular}{llll}
\hline \hline Value of $A_{2}$ of $E_{1}$ & Notation & $\begin{array}{l}\text { Ranking of the method } \\
\text { of Xu et al. (2019) }\end{array}$ & $\begin{array}{l}\text { Ranking of the proposed } \\
\text { method }\end{array}$ \\
\hline$<0.90,0.07,0.03>$ & $\mathrm{A}$ & $E_{1}>E_{3}>E_{5}>E_{4}>E_{2}$ & $E_{1}>E_{3}>E_{5}>E_{4}>E_{2}$ \\
$<0.80,0.07,0.03>$ & $\mathrm{B}$ & $E_{1}>E_{3}>E_{5}>E_{4}>E_{2}$ & $E_{1}>E_{3}>E_{5}>E_{4}>E_{2}$ \\
$<0.70,0.07,0.03>$ & $\mathrm{C}$ & $E_{1}>E_{3}>E_{5}>E_{4}>E_{2}$ & $E_{1}>E_{3}>E_{5}>E_{4}>E_{2}$ \\
$<0.60,0.07,0.03>$ & $\mathrm{D}$ & $E_{1}>E_{3}>E_{5}>E_{4}>E_{2}$ & $E_{1}>E_{3}>E_{5}>E_{4}>E_{2}$ \\
$<0.50,0.07,0.03>$ & $\mathrm{E}$ & $E_{1}>E_{3}>E_{5}>E_{4}>E_{2}$ & $E_{1}>E_{3}>E_{5}>E_{4}>E_{2}$
\end{tabular}




\begin{tabular}{llll}
$<0.40,0.07,0.03>$ & $\mathrm{F}$ & $E_{1}>E_{3}>E_{5}>E_{4}>E_{2}$ & $E_{1}>E_{3}>E_{5}>E_{4}>E_{2}$ \\
$<0.30,0.07,0.03>$ & $\mathrm{G}$ & $E_{3}>E_{5}>E_{1}>E_{4}>E_{2}$ & $E_{1}>E_{3}>E_{5}>E_{4}>E_{2}$ \\
$<0.20,0.07,0.03>$ & $\mathrm{H}$ & $E_{3}>E_{5}>E_{1}>E_{4}>E_{2}$ & $E_{3}>E_{5}>E_{1}>E_{4}>E_{2}$ \\
$<0.10,0.07,0.03>$ & $\mathrm{I}$ & $E_{3}>E_{5}>E_{4}>E_{1}>E_{2}$ & $E_{3}>E_{5}>E_{4}>E_{1}>E_{2}$ \\
$<0.01,0.07,0.03>$ & $\mathrm{J}$ & $E_{3}>E_{5}>E_{4}>E_{1}>E_{2}$ & $E_{3}>E_{5}>E_{4}>E_{1}>E_{2}$ \\
\hline \hline
\end{tabular}
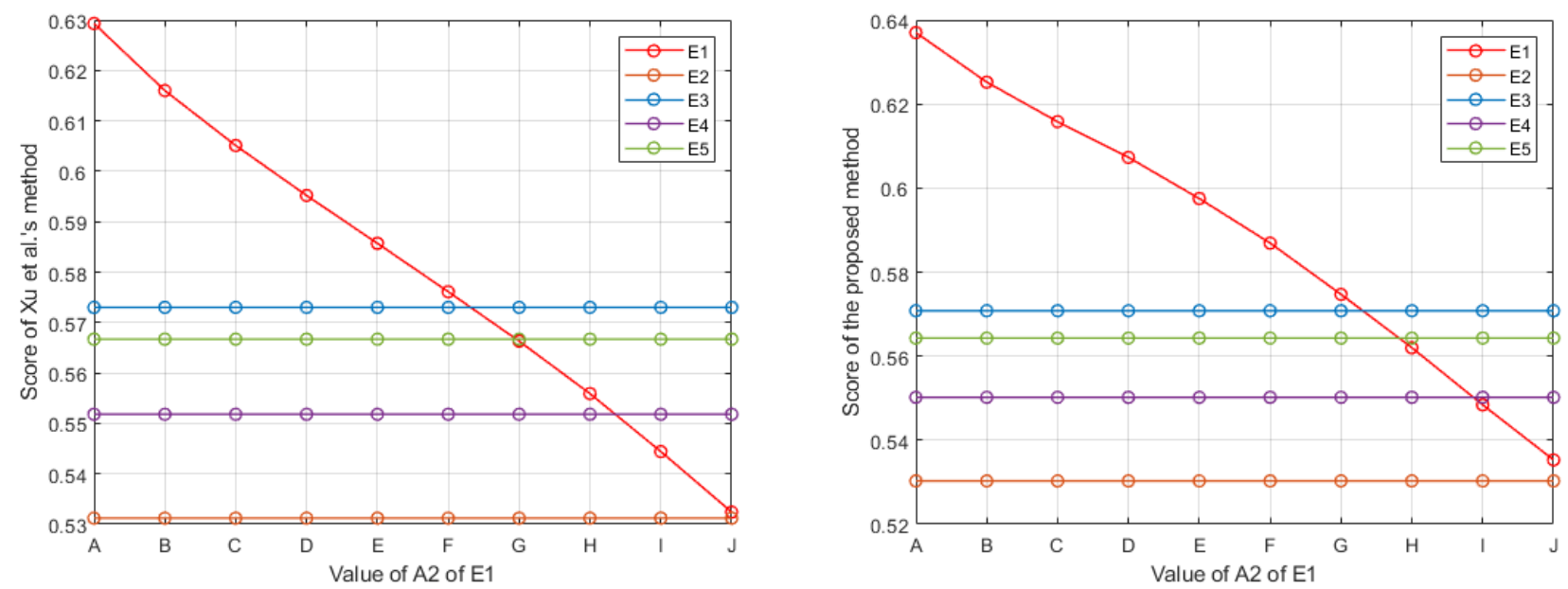

Figure 4. The change of score value and ranking of $E_{1}$ in the additional quantitative comparison.

From Table 6 and Figure 4, the results of both methods fit the conjecture. This demonstrates the effectiveness of the proposed method indirectly. In addition, the place of $E_{1}$ in the rankings of the method of Xu et al. (2019) descends faster than the place of $E_{1}$ in the rankings of the proposed method, which shows the capability to reduce the negative impact of biased attribute values intuitively.

As can be summarised from the comparisons above, the main advantages of the proposed method over the existing methods is providing the flexibility in the aggregation of picture fuzzy information and the consideration of the interactions among attributes and the capability to reduce the negative impact of some biased attribute values.

\section{Conclusion}

In the present paper, a PFAPMSM operator and a PFAPWMSM operator have been proposed to address the MADM problems based on PFNs. The formal definitions and the general expressions of these AOs have been presented. Their properties and special cases have been explored. The specific expressions of the two AOs have been established applying the operational rules of PFNs based on four families of ATTs. Using the established specific PFAPWMSM operators, a method to solve the PFNs based MADM problems has been proposed. The paper has also introduced a numerical example to illustrate the proposed method and carried out a set of comparisons to demonstrate its features, feasibility, and effectiveness. The comparison results show that the method is feasible and effective that provides the flexibility in aggregation of values of attributes, the generality in consideration of interactions among attributes, and the capability to reduce the negative impact of extreme values of attributes. Future work will focus especially on extending the proposed method from the aspects of dealing with more complex interactions among attributes and applying the method in solving practical MADM problems based on PFNs.

\section{Appendixes}

\section{A. Proof of Theorem 1}

\section{Proof:}

(1) The following equations are successively derived from the operational rules in Eq.s (1), (2), (3), and (4)

$$
\begin{aligned}
& \left(n \omega_{i_{h}}\right) \alpha_{i_{h}}=\left\langle\psi^{-1}\left(\left(n \omega_{i_{h}}\right) \psi\left(\mu_{i_{h}}\right)\right), \varphi^{-1}\left(\left(n \omega_{i_{h}}\right) \varphi\left(\eta_{i_{h}}\right)\right), \varphi^{-1}\left(\left(n \omega_{i_{h}}\right) \varphi\left(v_{i_{h}}\right)\right)\right\rangle \\
& \bigotimes_{h=1}^{k}\left(\left(n \omega_{i_{h}}\right) \alpha_{i_{h}}\right)=\left\langle\varphi^{-1}\left(\sum_{h=1}^{k} \varphi\left(\psi^{-1}\left(\left(n \omega_{i_{h}}\right) \psi\left(\mu_{i_{h}}\right)\right)\right)\right), \psi^{-1}\left(\sum_{h=1}^{k} \psi\left(\varphi^{-1}\left(\left(n \omega_{i_{h}}\right) \varphi\left(\eta_{i_{h}}\right)\right)\right)\right), \psi^{-1}\left(\sum_{h=1}^{k} \psi\left(\varphi^{-1}\left(\left(n \omega_{i_{h}}\right) \varphi\left(v_{i_{h}}\right)\right)\right)\right)\right\rangle
\end{aligned}
$$




$$
\begin{aligned}
& \bigoplus_{1 \leq i_{1}<\ldots<i_{k} \leq n} \bigotimes_{h=1}^{k}\left(\left(n \omega_{i_{h}}\right) \alpha_{i_{h}}\right)=\left\langle\psi ^ { - 1 } \left(\sum_{1 \leq i_{1}<\ldots<i_{k} \leq n} \psi\left(\varphi^{-1}\left(\sum_{h=1}^{k} \varphi\left(\psi^{-1}\left(\left(n \omega_{i_{h}}\right) \psi\left(\mu_{i_{h}}\right)\right)\right)\right)\right),\right.\right. \\
& \varphi^{-1}\left(\sum_{1 \leq i_{1}<\ldots<i_{k} \leq n} \varphi\left(\psi^{-1}\left(\sum_{h=1}^{k} \psi\left(\varphi^{-1}\left(\left(n \omega_{i_{h}}\right) \varphi\left(\eta_{i_{h}}\right)\right)\right)\right)\right)\right) \text {, } \\
& \left.\varphi^{-1}\left(\sum_{1 \leq i_{1}<\ldots<i_{k} \leq n} \varphi\left(\psi^{-1}\left(\sum_{h=1}^{k} \psi\left(\varphi^{-1}\left(\left(n \omega_{i_{h}}\right) \varphi\left(v_{i_{h}}\right)\right)\right)\right)\right)\right)\right\rangle \\
& \frac{1}{C_{n}^{k}} \bigoplus_{1 \leq i_{1}<\ldots<i_{k} \leq n} \bigotimes_{h=1}^{k}\left(\left(n \omega_{i_{h}}\right) \alpha_{i_{h}}\right)=\left\langle\psi ^ { - 1 } \left(\frac{1}{C_{n}^{k}} \sum_{1 \leq i_{1}<\ldots<i_{k} \leq n} \psi\left(\varphi^{-1}\left(\sum_{h=1}^{k} \varphi\left(\psi^{-1}\left(\left(n \omega_{i_{h}}\right) \psi\left(\mu_{i_{h}}\right)\right)\right)\right)\right),\right.\right. \\
& \varphi^{-1}\left(\frac{1}{C_{n}^{k}} \sum_{1 \leq i_{1}<\ldots<i_{k} \leq n} \varphi\left(\psi^{-1}\left(\sum_{h=1}^{k} \psi\left(\varphi^{-1}\left(\left(n \omega_{i_{h}}\right) \varphi\left(\eta_{i_{h}}\right)\right)\right)\right)\right),\right. \\
& \left.\varphi^{-1}\left(\frac{1}{C_{n}^{k}} \sum_{1 \leq i_{1}<\ldots<i_{k} \leq n} \varphi\left(\psi^{-1}\left(\sum_{h=1}^{k} \psi\left(\varphi^{-1}\left(\left(n \omega_{i_{h}}\right) \varphi\left(v_{i_{h}}\right)\right)\right)\right)\right)\right)\right\rangle \\
& \left(\frac{1}{C_{n}^{k}} \bigoplus_{1 \leq i_{1}<\ldots<i_{k} \leq n} \bigotimes_{h=1}^{k}\left(\left(n \omega_{i_{h}}\right) \alpha_{i_{h}}\right)\right)^{1 / k}=\left\langle\varphi^{-1}\left(\frac{1}{k} \varphi\left(\psi^{-1}\left(\frac{1}{C_{n}^{k}} \sum_{1 \leq i_{1}<\ldots<i_{k} \leq n} \psi\left(\varphi^{-1}\left(\sum_{h=1}^{k} \varphi\left(\psi^{-1}\left(\left(n \omega_{i_{h}}\right) \psi\left(\mu_{i_{h}}\right)\right)\right)\right)\right)\right)\right)\right),\right. \\
& \psi^{-1}\left(\frac{1}{k} \psi\left(\varphi^{-1}\left(\frac{1}{C_{n}^{k}} \sum_{1 \leq i_{1}<\ldots<i_{k} \leq n} \varphi\left(\psi^{-1}\left(\sum_{h=1}^{k} \psi\left(\varphi^{-1}\left(\left(n \omega_{i_{h}}\right) \varphi\left(\eta_{i_{h}}\right)\right)\right)\right)\right)\right)\right),\right. \\
& \psi^{-1}\left(\frac{1}{k} \psi\left(\varphi^{-1}\left(\frac{1}{C_{n}^{k}} \sum_{1 \leq i_{1}<\ldots<i_{k} \leq n} \varphi\left(\psi^{-1}\left(\sum_{h=1}^{k} \psi\left(\varphi^{-1}\left(\left(n \omega_{i_{h}}\right) \varphi\left(v_{i_{h}}\right)\right)\right)\right)\right)\right)\right)\right)
\end{aligned}
$$

(2) The proof of "PFAPMSM" $\left(\mathrm{P}^{(k)}\left(\alpha_{1}, \alpha_{2}, \ldots, \alpha_{n}\right)\right.$ is a PFN" is equivalent to the proof of " $0 \leq \mu \leq 1,0 \leq \eta \leq 1,0 \leq v \leq 1$, and 0 $\leq \mu+\eta+v \leq 1$ ". The following is the proof of " $0 \leq \mu \leq 1$ ":

1) According to the conditions $0 \leq \mu_{i} \leq 1, \varphi(t)$ and $\varphi^{-1}(t)$ are monotonically decreasing, and $\psi(t)$ and $\psi^{-1}(t)$ are monotonically increasing, the following inequalities are successively derived

$$
\begin{aligned}
& \left(n \omega_{i_{h}}\right) \psi(0) \leq\left(n \omega_{i_{h}}\right) \psi\left(\mu_{i_{h}}\right) \leq\left(n \omega_{i_{h}}\right) \psi(1) \\
& \psi^{-1}\left(\left(n \omega_{i_{h}}\right) \psi(0)\right) \leq \psi^{-1}\left(\left(n \omega_{i_{h}}\right) \psi\left(\mu_{i_{h}}\right)\right) \leq \psi^{-1}\left(\left(n \omega_{i_{h}}\right) \psi(1)\right) \\
& \sum_{h=1}^{k} \varphi\left(\psi^{-1}\left(\left(n \omega_{i_{h}}\right) \psi(0)\right)\right) \geq \sum_{h=1}^{k} \varphi\left(\psi^{-1}\left(\left(n \omega_{i_{h}}\right) \psi\left(\mu_{i_{h}}\right)\right)\right) \geq \sum_{h=1}^{k} \varphi\left(\psi^{-1}\left(\left(n \omega_{i_{h}}\right) \psi(1)\right)\right) \\
& \varphi^{-1}\left(\sum_{h=1}^{k} \varphi\left(\psi^{-1}\left(\left(n \omega_{i_{h}}\right) \psi(0)\right)\right)\right) \leq \varphi^{-1}\left(\sum_{h=1}^{k} \varphi\left(\psi^{-1}\left(\left(n \omega_{i_{h}}\right) \psi\left(\mu_{i_{h}}\right)\right)\right)\right) \leq \varphi^{-1}\left(\sum_{h=1}^{k} \varphi\left(\psi^{-1}\left(\left(n \omega_{i_{h}}\right) \psi(1)\right)\right)\right) \\
& \frac{1}{C_{n}^{k}} \sum_{1 \leq i_{1}<\ldots<i_{k} \leq n} \psi\left(\varphi^{-1}\left(\sum_{h=1}^{k} \varphi\left(\psi^{-1}\left(\left(n \omega_{i_{h}}\right) \psi(0)\right)\right)\right)\right) \leq \frac{1}{C_{n}^{k}} \sum_{1 \leq i_{1}<\ldots<i_{k} \leq n} \psi\left(\varphi^{-1}\left(\sum_{h=1}^{k} \varphi\left(\psi^{-1}\left(\left(n \omega_{i_{h}}\right) \psi\left(\mu_{i_{h}}\right)\right)\right)\right) \leq\right. \\
& \frac{1}{C_{n}^{k}} \sum_{1 \leq i_{1}<\ldots<i_{k} \leq n} \psi\left(\varphi^{-1}\left(\sum_{h=1}^{k} \varphi\left(\psi^{-1}\left(\left(n \omega_{i_{h}}\right) \psi(1)\right)\right)\right)\right)
\end{aligned}
$$

Since

$$
\sum_{1 \leq i_{1}<\ldots<i_{k} \leq n} \sum_{h=1}^{k}\left(n \omega_{i_{h}}\right)=n \sum_{1 \leq i_{1}<\ldots<i_{k} \leq n}\left(\omega_{i_{1}}+\omega_{i_{2}}+\ldots+\omega_{i_{k}}\right)=n \frac{1}{n}=1
$$

The following inequalities are successively obtained

$$
\begin{aligned}
& \psi\left(\varphi^{-1}(k \varphi(0))\right) \leq \frac{1}{C_{n}^{k}} \sum_{1 \leq i_{1}<\ldots<i_{k} \leq n} \psi\left(\varphi^{-1}\left(\sum_{h=1}^{k} \varphi\left(\psi^{-1}\left(\left(n \omega_{i_{h}}\right) \psi\left(\mu_{i_{h}}\right)\right)\right)\right) \leq \psi\left(\varphi^{-1}(k \varphi(1))\right)\right. \\
& \varphi^{-1}(k \varphi(0)) \leq \psi^{-1}\left(\frac{1}{C_{n}^{k}} \sum_{1 \leq i_{1}<\ldots<i_{k} \leq n} \psi\left(\varphi^{-1}\left(\sum_{h=1}^{k} \varphi\left(\psi^{-1}\left(\left(n \omega_{i_{h}}\right) \psi\left(\mu_{i_{h}}\right)\right)\right)\right)\right)\right) \leq \varphi^{-1}(k \varphi(1))
\end{aligned}
$$




$$
\begin{aligned}
& \varphi(0) \geq \frac{1}{k} \varphi\left(\psi^{-1}\left(\frac{1}{C_{n}^{k}} \sum_{1 \leq i_{1}<\ldots<i_{k} \leq n} \psi\left(\varphi^{-1}\left(\sum_{h=1}^{k} \varphi\left(\psi^{-1}\left(\left(n \omega_{i_{h}}\right) \psi\left(\mu_{i_{h}}\right)\right)\right)\right)\right)\right)\right) \geq \varphi(1) \\
& 0 \leq \varphi^{-1}\left(\frac{1}{k} \varphi\left(\psi^{-1}\left(\frac{1}{C_{n}^{k}} \sum_{1 \leq i_{1}<\ldots<i_{k} \leq n} \psi\left(\varphi^{-1}\left(\sum_{h=1}^{k} \varphi\left(\psi^{-1}\left(\left(n \omega_{i_{h}}\right) \psi\left(\mu_{i_{h}}\right)\right)\right)\right)\right)\right)\right) \leq 1\right.
\end{aligned}
$$

That is $0 \leq \mu \leq 1$. " $0 \leq \eta \leq 1$ " and " $0 \leq v \leq 1$ " can be proved in a similar way.

2) The following is the proof of " $0 \leq \mu+\eta+v \leq 1$ ":

Since $0 \leq \mu \leq 1,0 \leq \eta \leq 1$, and $0 \leq v \leq 1$, it is obtained that $0 \leq \mu+\eta+v \leq 3$. According to the definition of a PFN in Def. 1 , it is further obtained that $\mu_{i_{h}}+\eta_{i_{h}}+v_{i_{h}} \leq 1$ and $\mu_{i_{h}} \leq 1-\left(\eta_{i_{h}}+v_{i_{h}}\right)$.

According to the conditions $\varphi(t)$ and $\varphi^{-1}(t)$ are monotonically decreasing, $\psi(t)$ and $\psi^{-1}(t)$ are monotonically increasing, $\psi(1-t)=\varphi(t), \psi^{-1}(t)=1-\varphi^{-1}(t), \varphi(1-t)=\psi(t)$, and $\varphi^{-1}(t)=1-\psi^{-1}(t)$, the following inequalities are successively derived

$$
\begin{aligned}
& \left(n \omega_{i_{h}}\right) \psi\left(\mu_{i_{h}}\right) \leq\left(n \omega_{i_{h}}\right) \psi\left(1-\left(\eta_{i_{h}}+v_{i_{h}}\right)\right) \\
& \left(n \omega_{i_{h}}\right) \psi\left(\mu_{i_{h}}\right) \leq\left(n \omega_{i_{h}}\right) \varphi\left(\eta_{i_{h}}+v_{i_{h}}\right) \\
& \varphi\left(\eta_{i_{h}}+v_{i_{h}}\right) \leq 2 \varphi\left(\eta_{i_{h}}+v_{i_{h}}\right) \leq \varphi\left(\eta_{i_{h}}\right)+\varphi\left(v_{i_{h}}\right) \\
& \left(n \omega_{i_{h}}\right) \psi\left(\mu_{i_{h}}\right) \leq\left(n \omega_{i_{h}}\right) \varphi\left(\eta_{i_{h}}+v_{i_{h}}\right) \leq\left(n \omega_{i_{h}}\right)\left(\varphi\left(\eta_{i_{h}}\right)+\varphi\left(v_{i_{h}}\right)\right) \\
& \psi^{-1}\left(\left(n \omega_{i_{h}}\right) \psi\left(\mu_{i_{h}}\right)\right) \leq \psi^{-1}\left(\left(n \omega_{i_{h}}\right)\left(\varphi\left(\eta_{i_{h}}\right)+\varphi\left(v_{i_{h}}\right)\right)\right) \\
& \psi^{-1}\left(\left(n \omega_{i_{h}}\right) \psi\left(\mu_{i_{h}}\right)\right) \leq 1-\varphi^{-1}\left(\left(n \omega_{i_{h}}\right)\left(\varphi\left(\eta_{i_{h}}\right)+\varphi\left(v_{i_{h}}\right)\right)\right) \\
& \sum_{h=1}^{k} \varphi\left(\psi^{-1}\left(\left(n \omega_{i_{h}}\right) \psi\left(\mu_{i_{h}}\right)\right)\right) \geq \sum_{h=1}^{k} \varphi\left(1-\varphi^{-1}\left(\left(n \omega_{i_{h}}\right)\left(\varphi\left(\eta_{i_{h}}\right)+\varphi\left(v_{i_{h}}\right)\right)\right)\right) \\
& \sum_{h=1}^{k} \varphi\left(\psi^{-1}\left(\left(n \omega_{i_{h}}\right) \psi\left(\mu_{i_{h}}\right)\right)\right) \geq \sum_{h=1}^{k} \psi\left(\varphi^{-1}\left(\left(n \omega_{i_{h}}\right)\left(\varphi\left(\eta_{i_{h}}\right)+\varphi\left(v_{i_{h}}\right)\right)\right)\right) \\
& \varphi^{-1}\left(\sum_{h=1}^{k} \varphi\left(\psi^{-1}\left(\left(n \omega_{i_{h}}\right) \psi\left(\mu_{i_{h}}\right)\right)\right)\right) \leq \varphi^{-1}\left(\sum_{h=1}^{k} \psi\left(\varphi^{-1}\left(\left(n \omega_{i_{h}}\right)\left(\varphi\left(\eta_{i_{h}}\right)+\varphi\left(v_{i_{h}}\right)\right)\right)\right)\right) \\
& \varphi^{-1}\left(\sum_{h=1}^{k} \varphi\left(\psi^{-1}\left(\left(n \omega_{i_{h}}\right) \psi\left(\mu_{i_{h}}\right)\right)\right)\right) \leq 1-\psi^{-1}\left(\sum_{h=1}^{k} \psi\left(\varphi^{-1}\left(\left(n \omega_{i_{h}}\right)\left(\varphi\left(\eta_{i_{h}}\right)+\varphi\left(v_{i_{h}}\right)\right)\right)\right)\right) \\
& \frac{1}{C_{n}^{k}} \sum_{1 \leq i_{1}<\ldots<i_{k} \leq n} \psi\left(\varphi^{-1}\left(\sum_{h=1}^{k} \varphi\left(\psi^{-1}\left(\left(n \omega_{i_{h}}\right) \psi\left(\mu_{i_{h}}\right)\right)\right)\right)\right) \leq \frac{1}{C_{n}^{k}} \sum_{1 \leq i_{1}<\ldots<i_{k} \leq n} \psi\left(1-\psi^{-1}\left(\sum_{h=1}^{k} \psi\left(\varphi^{-1}\left(\left(n \omega_{i_{h}}\right)\left(\varphi\left(\eta_{i_{h}}\right)+\varphi\left(v_{i_{h}}\right)\right)\right)\right)\right)\right) \\
& \frac{1}{C_{n}^{k}} \sum_{1 \leq i_{1}<\ldots<i_{k} \leq n} \psi\left(\varphi^{-1}\left(\sum_{h=1}^{k} \varphi\left(\psi^{-1}\left(\left(n \omega_{i_{h}}\right) \psi\left(\mu_{i_{h}}\right)\right)\right)\right) \leq \frac{1}{C_{n}^{k}} \sum_{1 \leq i_{1}<\ldots<i_{k} \leq n} \varphi\left(\psi^{-1}\left(\sum_{h=1}^{k} \psi\left(\varphi^{-1}\left(\left(n \omega_{i_{h}}\right)\left(\varphi\left(\eta_{i_{h}}\right)+\varphi\left(v_{i_{h}}\right)\right)\right)\right)\right)\right)\right. \\
& \psi^{-1}\left(\frac{1}{C_{n}^{k}} \sum_{1 \leq i_{1}<\ldots<i_{k} \leq n} \psi\left(\varphi^{-1}\left(\sum_{h=1}^{k} \varphi\left(\psi^{-1}\left(\left(n \omega_{i_{h}}\right) \psi\left(\mu_{i_{h}}\right)\right)\right)\right)\right) \leq \psi^{-1}\left(\frac{1}{C_{n}^{k}} \sum_{1 \leq i_{1}<\ldots<i_{k} \leq n} \varphi\left(\psi^{-1}\left(\sum_{h=1}^{k} \psi\left(\varphi^{-1}\left(\left(n \omega_{i_{h}}\right)\left(\varphi\left(\eta_{i_{h}}\right)+\varphi\left(v_{i_{h}}\right)\right)\right)\right)\right)\right)\right.\right. \\
& \psi^{-1}\left(\frac{1}{C_{n}^{k}} \sum_{1 \leq i_{1}<\ldots<i_{k} \leq n} \psi\left(\varphi^{-1}\left(\sum_{h=1}^{k} \varphi\left(\psi^{-1}\left(\left(n \omega_{i_{h}}\right) \psi\left(\mu_{i_{h}}\right)\right)\right)\right)\right) \leq\right. \\
& 1-\varphi^{-1}\left(\frac{1}{C_{n}^{k}} \sum_{1 \leq i_{1}<\ldots<i_{k} \leq n} \varphi\left(\psi^{-1}\left(\sum_{h=1}^{k} \psi\left(\varphi^{-1}\left(\left(n \omega_{i_{h}}\right)\left(\varphi\left(\eta_{i_{h}}\right)+\varphi\left(v_{i_{h}}\right)\right)\right)\right)\right)\right)\right. \\
& \frac{1}{k} \varphi\left(\psi^{-1}\left(\frac{1}{C_{n}^{k}} \sum_{1 \leq i_{1}<\ldots<i_{k} \leq n} \psi\left(\varphi^{-1}\left(\sum_{h=1}^{k} \varphi\left(\psi^{-1}\left(\left(n \omega_{i_{h}}\right) \psi\left(\mu_{i_{h}}\right)\right)\right)\right)\right)\right) \geq\right. \\
& \frac{1}{k} \varphi\left(1-\varphi^{-1}\left(\frac{1}{C_{n}^{k}} \sum_{1 \leq i_{1}<\ldots<i_{k} \leq n} \varphi\left(\psi^{-1}\left(\sum_{h=1}^{k} \psi\left(\varphi^{-1}\left(\left(n \omega_{i_{h}}\right)\left(\varphi\left(\eta_{i_{h}}\right)+\varphi\left(v_{i_{h}}\right)\right)\right)\right)\right)\right)\right)\right.
\end{aligned}
$$

18 


$$
\begin{aligned}
& \frac{1}{k} \varphi\left(\psi^{-1}\left(\frac{1}{C_{n}^{k}} \sum_{1 \leq i_{1}<\ldots<i_{k} \leq n} \psi\left(\varphi^{-1}\left(\sum_{h=1}^{k} \varphi\left(\psi^{-1}\left(\left(n \omega_{i_{h}}\right) \psi\left(\mu_{i_{h}}\right)\right)\right)\right)\right)\right)\right) \geq \\
& \frac{1}{k} \psi\left(\varphi^{-1}\left(\frac{1}{C_{n}^{k}} \sum_{1 \leq i_{1}<\ldots<i_{k} \leq n} \varphi\left(\psi^{-1}\left(\sum_{h=1}^{k} \psi\left(\varphi^{-1}\left(\left(n \omega_{i_{h}}\right)\left(\varphi\left(\eta_{i_{h}}\right)+\varphi\left(v_{i_{h}}\right)\right)\right)\right)\right)\right)\right)\right. \\
& \varphi^{-1}\left(\frac{1}{k} \varphi\left(\psi^{-1}\left(\frac{1}{C_{n}^{k}} \sum_{1 \leq i_{1}<\ldots<i_{k} \leq n} \psi\left(\varphi^{-1}\left(\sum_{h=1}^{k} \varphi\left(\psi^{-1}\left(\left(n \omega_{i_{h}}\right) \psi\left(\mu_{i_{h}}\right)\right)\right)\right)\right)\right)\right) \leq\right. \\
& \varphi^{-1}\left(\frac{1}{k} \psi\left(\varphi^{-1}\left(\frac{1}{C_{n}^{k}} \sum_{1 \leq i_{1}<\ldots<i_{k} \leq n} \varphi\left(\psi^{-1}\left(\sum_{h=1}^{k} \psi\left(\varphi^{-1}\left(\left(n \omega_{i_{h}}\right)\left(\varphi\left(\eta_{i_{h}}\right)+\varphi\left(v_{i_{h}}\right)\right)\right)\right)\right)\right)\right)\right)\right) \\
& \varphi^{-1}\left(\frac{1}{k} \varphi\left(\psi^{-1}\left(\frac{1}{C_{n}^{k}} \sum_{1 \leq i_{1}<\ldots<i_{k} \leq n} \psi\left(\varphi^{-1}\left(\sum_{h=1}^{k} \varphi\left(\psi^{-1}\left(\left(n \omega_{i_{h}}\right) \psi\left(\mu_{i_{h}}\right)\right)\right)\right)\right)\right)\right) \leq\right. \\
& 1-\psi^{-1}\left(\frac{1}{k} \psi\left(\varphi^{-1}\left(\frac{1}{C_{n}^{k}} \sum_{1 \leq i_{1}<\ldots<i_{k} \leq n} \varphi\left(\psi^{-1}\left(\sum_{h=1}^{k} \psi\left(\varphi^{-1}\left(\left(n \omega_{i_{h}}\right)\left(\varphi\left(\eta_{i_{h}}\right)+\varphi\left(v_{i_{h}}\right)\right)\right)\right)\right)\right)\right)\right) \leq\right. \\
& 1-\left(\begin{array}{l}
\psi^{-1}\left(\frac{1}{k} \psi\left(\varphi^{-1}\left(\frac{1}{C_{n}^{k}} \sum_{1 \leq i_{1}<\ldots<i_{k} \leq n} \varphi\left(\psi^{-1}\left(\sum_{h=1}^{k} \psi\left(\varphi^{-1}\left(\left(n \omega_{i_{h}}\right)\left(\varphi\left(\eta_{i_{h}}\right)\right)\right)\right)\right)\right)\right)\right)+\right. \\
\psi^{-1}\left(\frac{1}{k} \psi\left(\varphi^{-1}\left(\frac{1}{C_{n}^{k}} \sum_{1 \leq i_{1}<\ldots<i_{k} \leq n} \varphi\left(\psi^{-1}\left(\sum_{h=1}^{k} \psi\left(\varphi^{-1}\left(\left(n \omega_{i_{h}}\right)\left(\varphi\left(v_{i_{h}}\right)\right)\right)\right)\right)\right)\right)\right)\right)
\end{array}\right)
\end{aligned}
$$

That is, $\mu+\eta+v \leq 1$.

Since $0 \leq \mu+\eta+v \leq 3$ and $\mu+\eta+v \leq 1$ have been proved, $0 \leq \mu+\eta+v \leq 1$ can be obtained.

\section{B. Proof of Theorem 2}

Proof:

Since $\alpha_{i}=\alpha=\left\langle\mu_{\alpha}, \eta_{\alpha}, v_{\alpha}\right\rangle$ for all $i=1,2, \ldots, n, d\left(\alpha_{i}, \alpha_{j}\right)=0$ for all $j=1,2, \ldots, n$ and $j \neq i$. Based on the expression of $T\left(\alpha_{p}\right)$ in Def. 9 , it is obtained that

$$
n \omega_{i_{h}}=n\left(\sum_{1 \leq i_{1}<\ldots<i_{k} \leq n} \sum_{h=1}^{k}\left(1+T\left(\alpha_{i_{h}}\right)\right)\right) / \sum_{j=1}^{n}\left(1+T\left(\alpha_{j}\right)\right)=n(1+(n-1)) /(n(1+(n-1)))=1
$$

Based on this and Theorem 1, it can be obtained that

$$
\mu=\varphi^{-1}\left(\frac{1}{k} \varphi\left(\psi^{-1}\left(\frac{1}{C_{n}^{k}} \sum_{1 \leq i_{1}<\ldots<i_{k} \leq n} \psi\left(\varphi^{-1}\left(\sum_{h=1}^{k} \varphi\left(\mu_{i_{h}}\right)\right)\right)\right)\right)\right.
$$

Since $\mu_{i}=\mu_{\alpha}$, then

$$
\sum_{h=1}^{k} \varphi\left(\mu_{i_{h}}\right)=\sum_{h=1}^{k} \varphi\left(\mu_{\alpha}\right)=k \varphi\left(\mu_{\alpha}\right) \text { and } \varphi^{-1}\left(\sum_{h=1}^{k} \varphi\left(\mu_{i_{h}}\right)\right)=\varphi^{-1}\left(k \varphi\left(\mu_{\alpha}\right)\right)
$$

The following equations are successively obtained

$$
\begin{aligned}
& \frac{1}{C_{n}^{k}} \sum_{1 \leq i_{1}<\ldots<i_{k} \leq n} \psi\left(\varphi^{-1}\left(\sum_{h=1}^{k} \varphi\left(\mu_{i_{h}}\right)\right)\right)=\frac{1}{C_{n}^{k}} \sum_{1 \leq i_{1}<\ldots<i_{k} \leq n} \psi\left(\varphi^{-1}\left(k \varphi\left(\mu_{\alpha}\right)\right)\right)=\psi\left(\varphi^{-1}\left(k \varphi\left(\mu_{\alpha}\right)\right)\right) \\
& \psi^{-1}\left(\frac{1}{C_{n}^{k}} \sum_{1 \leq i_{1}<\ldots<i_{k} \leq n} \psi\left(\varphi^{-1}\left(\sum_{h=1}^{k} \varphi\left(\mu_{i_{h}}\right)\right)\right)\right)=\psi^{-1}\left(\psi\left(\varphi^{-1}\left(k \varphi\left(\mu_{\alpha}\right)\right)\right)\right)=\varphi^{-1}\left(k \varphi\left(\mu_{\alpha}\right)\right) \\
& \frac{1}{k} \varphi\left(\psi^{-1}\left(\frac{1}{C_{n}^{k}} \sum_{1 \leq i_{1}<\ldots<i_{k} \leq n} \psi\left(\varphi^{-1}\left(\sum_{h=1}^{k} \varphi\left(\mu_{i_{h}}\right)\right)\right)\right)=\frac{1}{k} \varphi\left(\varphi^{-1}\left(k \varphi\left(\mu_{\alpha}\right)\right)\right)=\varphi\left(\mu_{\alpha}\right)\right. \\
& \varphi^{-1}\left(\frac{1}{k} \varphi\left(\psi^{-1}\left(\frac{1}{C_{n}^{k}} \sum_{1 \leq i_{1}<\ldots<i_{k} \leq n} \psi\left(\varphi^{-1}\left(\sum_{h=1}^{k} \varphi\left(\mu_{i_{h}}\right)\right)\right)\right)\right)\right)=\varphi^{-1}\left(\varphi\left(\mu_{\alpha}\right)\right)=\mu_{\alpha}
\end{aligned}
$$




$$
\varphi^{-1}\left(\frac{1}{k} \varphi\left(\psi^{-1}\left(\frac{1}{C_{n}^{k}} \sum_{1 \leq i_{1}<\ldots<i_{k} \leq n} \psi\left(\varphi^{-1}\left(\sum_{h=1}^{k} \varphi\left(\psi^{-1}\left(\left(n \omega_{i_{h}}\right) \psi\left(\mu_{i_{h}}\right)\right)\right)\right)\right)\right)\right)\right)=\mu_{\alpha}
$$

This is $\mu=\mu_{\alpha}$. Similarly, it can be proved that $\eta=\eta_{\alpha}$ and $v=v_{\alpha}$. Thus, $\operatorname{PFAPMSM}^{(k)}\left(\alpha_{1}, \alpha_{2}, \ldots, \alpha_{n}\right)=\left\langle\mu_{\alpha}, \eta_{\alpha}, v_{\alpha}\right\rangle$.

\section{Proof of Theorem 3}

Proof:

It can be derived from the definition of the PFAPMSM operator that

$$
\left(\frac{1}{C_{n}^{k}} \bigoplus_{1 \leq i_{1}<\ldots<i_{k} \leq n} \bigotimes_{h=1}^{k}\left(\frac{n\left(1+T\left(\beta_{i_{h}}\right)\right)}{\sum_{j=1}^{n}\left(1+T\left(\beta_{j}\right)\right)} \beta_{i_{h}}\right)\right)^{1 / k}=\left(\frac{1}{C_{n}^{k}} \bigoplus_{1 \leq i_{1}<\ldots<i_{k} \leq n} \bigotimes_{h=1}^{k}\left(\frac{n\left(1+T\left(\alpha_{i_{h}}\right)\right)}{\sum_{j=1}^{n}\left(1+T\left(\alpha_{j}\right)\right)} \alpha_{i_{h}}\right)\right)^{1 / k}
$$

According to Def. 9, it is obtained that $\operatorname{PFAPMSM}^{(k)}\left(\beta_{1}, \beta_{2}, \ldots, \beta_{n}\right)=\operatorname{PFAPMSM}^{(k)}\left(\alpha_{1}, \alpha_{2}, \ldots, \alpha_{n}\right)$.

\section{Proof of Theorem 4}

Proof:

Based on Theorem 2, it can be obtained that $\operatorname{PFAPMSM} M^{(k)}\left(\alpha^{-}, \alpha^{-}, \ldots, \alpha^{-}\right)=\alpha^{-}, P F A P M S M^{(k)}\left(\alpha^{+}, \alpha^{+}, \ldots, \alpha^{+}\right)=\alpha^{+}$, and $n \xi_{i_{h}}=$ 1 for both $P F A P M S M^{(k)}\left(\alpha^{-}, \alpha^{-}, \ldots, \alpha^{-}\right)$and $P F A P M S M^{(k)}\left(\alpha^{+}, \alpha^{+}, \ldots, \alpha^{+}\right)$. According to the conditions $\mu^{-} \leq \mu_{i h} \leq \mu^{+}, \varphi(t)$ and $\varphi^{-1}(t)$ are monotonically decreasing, and $\psi(t)$ and $\psi^{-1}(t)$ are monotonically increasing, the following inequalities are successively derived

$$
\begin{aligned}
& \psi\left(\mu^{-}\right) \leq\left(n \omega_{i_{h}}\right) \psi\left(\mu_{i_{h}}\right) \leq \psi\left(\mu^{+}\right) \\
& \mu^{-} \leq \psi^{-1}\left(\left(n \omega_{i_{h}}\right) \psi\left(\mu_{i_{h}}\right)\right) \leq \mu^{+} \\
& k \varphi\left(\mu^{-}\right) \geq \sum_{h=1}^{k} \varphi\left(\psi^{-1}\left(\left(n \omega_{i_{h}}\right) \psi\left(\mu_{i_{h}}\right)\right)\right) \geq k \varphi\left(\mu^{+}\right) \\
& \varphi^{-1}\left(k \varphi\left(\mu^{-}\right)\right) \leq \varphi^{-1}\left(\sum_{h=1}^{k} \varphi\left(\psi^{-1}\left(\left(n \omega_{i_{h}}\right) \psi\left(\mu_{i_{h}}\right)\right)\right)\right) \leq \varphi^{-1}\left(k \varphi\left(\mu^{+}\right)\right) \\
& \psi\left(\varphi^{-1}\left(k \varphi\left(\mu^{-}\right)\right)\right) \leq \frac{1}{C_{n}^{k}} \sum_{1 \leq i_{1}<\ldots<i_{k} \leq n} \psi\left(\varphi^{-1}\left(\sum_{h=1}^{k} \varphi\left(\psi^{-1}\left(\left(n \omega_{i_{h}}\right) \psi\left(\mu_{i_{h}}\right)\right)\right)\right)\right) \leq \psi\left(\varphi^{-1}\left(k \varphi\left(\mu^{+}\right)\right)\right) \\
& \varphi^{-1}\left(k \varphi\left(\mu^{-}\right)\right) \leq \psi^{-1}\left(\frac{1}{C_{n}^{k}} \sum_{1 \leq i_{1}<\ldots<i_{k} \leq n} \psi\left(\varphi^{-1}\left(\sum_{h=1}^{k} \varphi\left(\psi^{-1}\left(\left(n \omega_{i_{h}}\right) \psi\left(\mu_{i_{h}}\right)\right)\right)\right)\right)\right) \leq \varphi^{-1}\left(k \varphi\left(\mu^{+}\right)\right) \\
& \varphi\left(\mu^{-}\right) \geq \frac{1}{k} \varphi\left(\psi^{-1}\left(\frac{1}{C_{n}^{k}} \sum_{1 \leq i_{1}<\ldots<i_{k} \leq n} \psi\left(\varphi^{-1}\left(\sum_{h=1}^{k} \varphi\left(\psi^{-1}\left(\left(n \omega_{i_{h}}\right) \psi\left(\mu_{i_{h}}\right)\right)\right)\right)\right)\right)\right) \geq \varphi\left(\mu^{+}\right) \\
& \mu^{-} \leq \varphi^{-1}\left(\frac{1}{k} \varphi\left(\psi^{-1}\left(\frac{1}{C_{n}^{k}} \sum_{1 \leq i_{1}<\ldots<i_{k} \leq n} \psi\left(\varphi^{-1}\left(\sum_{h=1}^{k} \varphi\left(\psi^{-1}\left(\left(n \omega_{i_{h}}\right) \psi\left(\mu_{i_{h}}\right)\right)\right)\right)\right)\right)\right) \leq \mu^{+}\right.
\end{aligned}
$$

That is $\mu^{-} \leq \mu \leq \mu^{+}$. Similarly, it can be proved that $\eta^{-} \geq \eta \geq \eta^{+}$and $v^{-} \geq v \geq v^{+}$.

According to Def. 2, it is obtained that

$\operatorname{PFAPMSM}^{(k)}\left(\alpha^{-}, \alpha^{-}, \ldots, \alpha^{-}\right) \leq \operatorname{PFAPMSM}^{(k)}\left(\alpha_{1}, \alpha_{2}, \ldots, \alpha_{n}\right) \leq \operatorname{PFAPMSM}^{(k)}\left(\alpha^{+}, \alpha^{+}, \ldots, \alpha^{+}\right)$

Therefore, $\alpha^{-} \leq$PFAPMSM $^{(k)}\left(\alpha_{1}, \alpha_{2}, \ldots, \alpha_{n}\right) \leq \alpha^{+}$.

\section{Data Availability}

The Java implementation code of all quantitative comparison methods and related data used to support the findings of this study have been deposited in the GitHub repository (https://github.com/YuchuChingQin/MADMAOsOfPFNs).

\section{Compliance with Ethical Standards}

Funding: This study was funded by the National Natural Science Foundation of PR China (No. 51765012 and No. 61562016) and the Key Laboratory Project of Guangxi (No. GIIP1805). 
Conflict of Interest: Yuchu Qin declares that he has no conflict of interest. Xiaolan Cui declares that she has no conflict of interest. Meifa Huang declares that he has no conflict of interest. Yanru Zhong declares that she has no conflict of interest. Zhemin Tang declares that he has no conflict of interest. Peizhi Shi declares that he has no conflict of interest.

Ethical Approval: This article does not contain any studies with human participants or animals performed by any of the authors.

\section{Reference}

Ai Z, Xu Z (2018) Multiple Definite Integrals of Intuitionistic Fuzzy Calculus and Isomorphic Mappings. IEEE Transactions on Fuzzy Systems 26(2): 670-680

Atanassov KT (1986) Intuitionistic fuzzy sets. Fuzzy Sets and Systems 20(1): 87-96

Bustince H, Barrenechea E, Pagola M, Fernandez J, Xu Z, Bedregal B, Montero J, Hagras H, Herrera F, De Baets B (2016) A historical account of types of fuzzy sets and their relationships. IEEE Transactions on Fuzzy Systems 24(1): 179-194

Castillo O, Cervantes L, Melin P, Pedrycz W (2019) A new approach to control of multivariable systems through a hierarchical aggregation of fuzzy controllers. Granular Computing 4(1): 1-13

Chen SM, Adam SI (2017) Adaptive fuzzy interpolation based on general representative values of polygonal fuzzy sets and the shift and modification techniques. Information Sciences 414: 147-157

Chen SM, Chang CH (2016) Fuzzy multiattribute decision making based on transformation techniques of intuitionistic fuzzy values and intuitionistic fuzzy geometric averaging operators. Information Sciences 352: 133-149

Chen SM, Chen SW (2014) Fuzzy forecasting based on two-factors second-order fuzzy-trend logical relationship groups and the probabilities of trends of fuzzy logical relationships. IEEE Transactions on Cybernetics 45(3): 391-403

Chen SM, Niou SJ (2011) Fuzzy multiple attributes group decision-making based on fuzzy preference relations. Expert Systems with Applications 38(4): 3865-3872

Chen SM, Ko YK, Chang YC, Pan JS (2009) Weighted fuzzy interpolative reasoning based on weighted increment transformation and weighted ratio transformation techniques. IEEE Transactions on Fuzzy Systems 17(6): 1412-1427

Chen SM, Chu HP, Sheu TW (2012) TAIEX forecasting using fuzzy time series and automatically generated weights of multiple factors. IEEE Transactions on Systems, Man, and Cybernetics-Part A: Systems and Humans 42(6): 1485-1495

Chen SM, Cheng SH, Lan TC (2016a) Multicriteria decision making based on the TOPSIS method and similarity measures between intuitionistic fuzzy values. Information Sciences 367: 279-295

Chen SM, Cheng SH, Chiou CH (2016b) Fuzzy multiattribute group decision making based on intuitionistic fuzzy sets and evidential reasoning methodology. Information Fusion 27: 215-227

Cuong BC (2014) Picture fuzzy sets. Journal of Computer Science and Cybernetics 30(4): 409-420

Dutta P (2019) Multi-criteria decision making under uncertainty via the operations of generalized intuitionistic fuzzy numbers. Granular Computing. https://doi.org/10.1007/s41066-019-00189-z

Dutta P, Saikia B (2019) Arithmetic operations on normal semi elliptic intuitionistic fuzzy numbers and their application in decision-making. Granular Computing. https://doi.org/10.1007/s41066-019-00175-5

Garg H (2017a) Novel intuitionistic fuzzy decision making method based on an improved operation laws and its application. Engineering Applications of Artificial Intelligence 60: 164-174

Garg H (2017b) Some picture fuzzy aggregation operators and their applications to multicriteria decision-making. Arabian Journal for Science and Engineering 42(12): 5275-5290

Garg H, Kumar K (2018) An advanced study on the similarity measures of intuitionistic fuzzy sets based on the set pair analysis theory and their application in decision making. Soft Computing 22(15): 4959-4970

Garg H, Kumar K (2019) Improved possibility degree method for ranking intuitionistic fuzzy numbers and their application in multiattribute decision-making. Granular Computing 4(2): 237-247

Hinde CJ, Patching RS, McCoy SA (2007) Inconsistent Intuitionistic Fuzzy Sets and Mass Assignment. In: Atanassov KT, Baczyński M, Drewniak J, Kacprzyk J, Krawczak M, Szmidt E, Wygralak M, Zadrożny S (Eds.) Developments in Fuzzy Sets, Intuitionistic Fuzzy Sets, Generalized Nets and Related Topics. Vol. I: Foundations, Warsaw: SRI PAS/IBS PAN, pp. $133-153$

Jamkhaneh EB, Garg H (2018) Some new operations over the generalized intuitionistic fuzzy sets and their application to decisionmaking process. Granular Computing 3(2): 111-122

Jana C, Senapati T, Pal M, Yager RR (2019) Picture fuzzy Dombi aggregation operators: Application to MADM process. Applied Soft Computing 74: 99-109

Ju Y, Ju D, Gonzalez EDS, Giannakis M, Wang A (2019) Study of site selection of electric vehicle charging station based on extended GRP method under picture fuzzy environment. Computers \& Industrial Engineering 135: 1271-1285

Khalil AM, Li SG, Garg H, Li H, Ma S (2019) New Operations on Interval-Valued Picture Fuzzy Set, Interval-Valued Picture Fuzzy Soft Set and Their Applications. IEEE Access 7: 51236-51253

Klement EP, Mesiar R, Pap E (2000) Triangular Norms. Dordrecht: Springer Netherlands 
Kumar K, Garg H (2018) Connection number of set pair analysis based TOPSIS method on intuitionistic fuzzy sets and their application to decision making. Applied Intelligence 48(8): 2112-2119

Lei Q, Xu Z (2016) Chain and substitution rules of intuitionistic fuzzy calculus. IEEE Transactions on Fuzzy Systems 24(3): $519-529$

Lei Q, Xu Z (2017) A unification of intuitionistic fuzzy calculus theories based on subtraction derivatives and division derivatives. IEEE Transactions on Fuzzy Systems 25(5): 1023-1040

Liang W, Zhao G, Luo S (2018) An integrated EDAS-ELECTRE method with picture fuzzy information for cleaner production evaluation in gold mines. IEEE Access 6: 65747-65759

Liao H, Xu Z (2014) Priorities of intuitionistic fuzzy preference relation based on multiplicative consistency. IEEE Transactions on Fuzzy Systems 22(6): 1669-1681

Liao H, Xu Z, Zeng XJ, Merigó JM (2015) Framework of group decision making with intuitionistic fuzzy preference information. IEEE Transactions on Fuzzy Systems 23(4): 1211-1227

Liu P, Chen SM (2017) Group decision making based on Heronian aggregation operators of intuitionistic fuzzy numbers. IEEE Transactions on Cybernetics 47(9): 2514-2530

Liu P, Chen SM, Wang P (2018) Multiple-Attribute Group Decision-Making Based on q-Rung Orthopair Fuzzy Power Maclaurin Symmetric Mean Operators. IEEE Transactions on Systems, Man, and Cybernetics: Systems. https://doi.org/10.1109/tsmc. 2018.2852948

Liu P, Chen SM, Wang Y (2020) Multiattribute group decision making based on intuitionistic fuzzy partitioned Maclaurin symmetric mean operators. Information Sciences 512: 830-854

Liu P, Tang G (2018) Some Intuitionistic Fuzzy Prioritized Interactive Einstein Choquet Operators and Their Application in Decision Making. IEEE Access 6: 72357-72371

Liu P, Liu X (2017) Multiattribute group decision making methods based on linguistic intuitionistic fuzzy power Bonferroni mean operators. Complexity 2017: 3571459

Liu P, Liu J (2018) Some q-rung orthopai fuzzy Bonferroni mean operators and their application to multi-attribute group decision making. International Journal of Intelligent Systems 33(2): 315-347

Liu P, Wang P (2018) Some q-rung orthopair fuzzy aggregation operators and their applications to multiple-attribute decision making. International Journal of Intelligent Systems 33(2): 259-280

Liu P, Wang P (2019) Multiple-attribute decision making based on Archimedean Bonferroni operators of q-rung orthopair fuzzy numbers. IEEE Transactions on Fuzzy Systems 27(5): 834-848

Maclaurin C (1729) A second letter to Martin Folkes, Esq.; Concerning the roots of equations, with the demonstration of other rules in algebra. Philosophical Transactions of the Royal Society of London 36(1): 59-96

Mahmood T, Ullah K, Khan Q, Jan N (2019) An approach toward decision-making and medical diagnosis problems using the concept of spherical fuzzy sets. Neural Computing and Applications 31(11): 7041-7053

Qin Y, Qi Q, Scott PJ, Jiang X (2019a) Multi-criteria group decision making based on Archimedean power partitioned Muirhead mean operators of q-rung orthopair fuzzy numbers. PLoS ONE 14(9): e0221759

Qin Y, Cui X, Huang M, Zhong Y, Tang Z, Shi P (2019b) Archimedean Muirhead Aggregation Operators of q-Rung Orthopair Fuzzy Numbers for Multicriteria Group Decision Making. Complexity 2019: 3103741

Qin Y, Cui X, Huang M, Zhong Y, Tang Z, Shi P (2020a) Linguistic Interval-Valued Intuitionistic Fuzzy Archimedean Power Muirhead Mean Operators for Multiattribute Group Decision-Making. Complexity 2020: 2373762

Qin Y, Qi Q, Shi P, Scott PJ, Jiang X (2020b) Linguistic interval-valued intuitionistic fuzzy Archimedean prioritised aggregation operators for multi-criteria decision making. Journal of Intelligent \& Fuzzy Systems 38(4): 4643-4666

Qin Y, Qi Q, Scott PJ, Jiang X (2020c) Multiple criteria decision making based on weighted Archimedean power partitioned Bonferroni aggregation operators of generalised orthopair membership grades. Soft Computing. https://doi.org/10.1007/s00500020-04676-3

Rani P, Jain D, Hooda DS (2019) Extension of intuitionistic fuzzy TODIM technique for multi-criteria decision making method based on shapley weighted divergence measure. Granular Computing 4(3): 407-420

Seikh MR, Mandal U (2019) Intuitionistic fuzzy Dombi aggregation operators and their application to multiple attribute decisionmaking. Granular Computing. https://doi.org/10.1007/s41066-019-00209-y

Singh P (2015) Correlation coefficients for picture fuzzy sets. Journal of Intelligent \& Fuzzy Systems 28(2): 591-604

Son LH (2016) Generalized picture distance measure and applications to picture fuzzy clustering. Applied Soft Computing 46: 284-295

Song Y, Fu Q, Wang YF, Wang X (2019) Divergence-based cross entropy and uncertainty measures of Atanassov's intuitionistic fuzzy sets with their application in decision making. Applied Soft Computing 84: 105703

Tan A, Shi S, Wu WZ, Li J, Pedrycz W (2020) Granularity and Entropy of Intuitionistic Fuzzy Information and Their Applications. IEEE Transactions on Cybernetics. https://doi.org/10.1109/tcyb.2020.2973379

Teng F, Liu Z, Liu P (2018) Some power Maclaurin symmetric mean aggregation operators based on Pythagorean fuzzy linguistic numbers and their application to group decision making. International Journal of Intelligent Systems 33(9): 1949-1985 
Thong PH (2016) A novel automatic picture fuzzy clustering method based on particle swarm optimization and picture composite cardinality. Knowledge-Based Systems 109: 48-60

Wang JQ, Zhang HY (2013) Multicriteria decision-making approach based on Atanassov's intuitionistic fuzzy sets with incomplete certain information on weights. IEEE Transactions on Fuzzy Systems 21(3): 510-515

Wang L, Zhang H, Wang J, Li L (2018a) Picture fuzzy normalized projection-based VIKOR method for the risk evaluation of construction project. Applied Soft Computing 64: 216-226

Wang L, Peng J, Wang J (2018b) A multi-criteria decision-making framework for risk ranking of energy performance contracting project under picture fuzzy environment. Journal of Cleaner Production 191: 105-118

Wei G (2017) Picture fuzzy aggregation operators and their application to multiple attribute decision making. Journal of Intelligent \& Fuzzy Systems 33(2): 713-724

Wei G (2018) Picture fuzzy Hamacher aggregation operators and their application to multiple attribute decision making. Fundamenta Informaticae 157(3): 271-320

Wei G, Lu M, Gao H (2018a) Picture fuzzy Heronian mean aggregation operators in multiple attribute decision making. International Journal of Knowledge-Based and Intelligent Engineering Systems 22(3): 167-175

Wei G, Alsaadi FE, Hayat T, Alsaedi A (2018b) Picture 2-tuple linguistic aggregation operators in multiple attribute decision making. Soft Computing 22(3): 989-1002

Xia M, Xu Z, Zhu B (2012) Some issues on intuitionistic fuzzy aggregation operators based on Archimedean t-conorm and t-norm. Knowledge-Based Systems 31: 78-88

Xu Y, Shang X, Wang J, Zhang R, Li W, Xing Y (2019) A method to multi-attribute decision making with picture fuzzy information based on Muirhead mean. Journal of Intelligent \& Fuzzy Systems 36(4): 3833-3849

Xu Z, Yager RR (2011) Intuitionistic fuzzy Bonferroni means. IEEE Transactions on Systems, Man and Cybernetics-Part B: Cybernetics 41(2): 568-578

Yager RR (2001) The Power Average Operator. IEEE Transactions on Systems, Man, and Cybernetics-Part A: Systems and Humans 31(6): 724-731

Zadeh LA (1965) Fuzzy Sets. Information and Control 8(3): 338-353

Zeng S, Chen SM, Kuo LW (2019) Multiattribute decision making based on novel score function of intuitionistic fuzzy values and modified VIKOR method. Information Sciences 488: 76-92

Zhang FW, Huang WW, Sun J, Liu ZD, Zhu YH, Li KT, Xu SH, Li Q (2019) Generalized fuzzy additive operators on intuitionistic fuzzy sets and interval-valued intuitionistic fuzzy sets and their application. IEEE Access 7: 45734-45743

Zhang H, Zhang R, Huang H, Wang J (2018) Some picture fuzzy Dombi Heronian mean operators with their application to multiattribute decision-making. Symmetry 10(11): 593

Zhang Z, Pedrycz W, (2017a) Models of mathematical programming for intuitionistic multiplicative preference relations. IEEE Transactions on Fuzzy Systems 25(4): 945-957

Zhang Z, Pedrycz W (2017b) Intuitionistic multiplicative group analytic hierarchy process and its use in multicriteria group decision-making. IEEE Transactions on Cybernetics 48(7): 1950-1962

Zhang Z, Pedrycz W (2018) Goal programming approaches to managing consistency and consensus for intuitionistic multiplicative preference relations in group decision making. IEEE Transactions on Fuzzy Systems 26(6): 3261-3275

Zhang Z, Chen SM, Wang C (2020) Group decision making with incomplete intuitionistic multiplicative preference relations. Information Sciences 516: 560-571

Zhong Y, Guo X, Gao H, Qin Y, Huang M, Luo X (2019a) A new multi-criteria decision-making method based on Pythagorean hesitant fuzzy Archimedean Muirhead mean operators. Journal of Intelligent \& Fuzzy Systems 37(4): 5551-5571

Zhong Y, Gao H, Guo X, Qin Y, Huang M, Luo X (2019b) Dombi power partitioned Heronian mean operators of q-rung orthopair fuzzy numbers for multiple attribute group decision making. PLoS ONE 14(10): e0222007 